\title{
Large-format, high-speed, X-ray pnCCDs combined with electron and ion imaging spectrometers in a multipurpose chamber for experiments at 4 th generation light sources
}

Lothar Strüder $^{\text {a,c,e,h }}$, Sascha Epp ${ }^{\text {a }}$, Daniel Rolles ${ }^{\mathrm{a}}$, Robert Hartmann ${ }^{\mathrm{b}, \mathrm{e}}$, Peter Holl ${ }^{\mathrm{b}, \mathrm{e}}$, Gerhard Lutz ${ }^{\mathrm{b}, \mathrm{e}}$, Heike Soltau $^{\text {b,e }}$, Rouven Eckart ${ }^{\text {b,e }}$, Christian Reich ${ }^{\text {b,e }}$, Klaus Heinzinger ${ }^{\text {b,e }}$, Christian Thamm ${ }^{\text {b,e }}$, Artem Rudenko ${ }^{a}$, Faton Krasniqi a , Kai-Uwe Kühnel ${ }^{i}$, Christian Bauer ${ }^{i}$, Claus-Dieter Schröter $^{\mathrm{i}}$, Robert Moshammer ${ }^{\mathrm{a}, \mathrm{i}}$, Simone Techert ${ }^{\mathrm{a}, \mathrm{m}}$, Danilo Miessner ${ }^{\mathrm{c}, \mathrm{e}}$, Matteo Porro $^{\mathrm{c}, \mathrm{e}}$, Olaf Hälker ${ }^{\mathrm{c}, \mathrm{e}}$, Norbert Meidinger $^{c, e}$, Nils Kimmel ${ }^{\mathrm{c}, \mathrm{e}, *}$, Robert Andritschke ${ }^{\mathrm{c}, \mathrm{e}}$, Florian Schopper ${ }^{\mathrm{c}, \mathrm{e}}$, Georg Weidenspointner $^{c, e}$, Alexander Ziegler ${ }^{\text {c,e }}$, Daniel Pietschner ${ }^{c, e}$, Sven Herrmann ${ }^{c, e}$, Ullrich Pietsch $^{\mathrm{h}}$, Albert Walenta ${ }^{\mathrm{h}}$, Wolfram Leitenberger ${ }^{\mathrm{h}}$, Christoph Bostedt ${ }^{\mathrm{f}}$, Thomas Möller ${ }^{\mathrm{f}}$, Daniela Rupp ${ }^{\mathrm{f}}$, Marcus Adolph ${ }^{\mathrm{f}}$, Heinz Graafsma ${ }^{\mathrm{g}}$, Helmut Hirsemann ${ }^{\mathrm{g}}$, Klaus Gärtner ${ }^{\mathrm{k}}$, Rainer Richter ${ }^{\text {d,e }}$, Lutz Foucar ${ }^{a}$, Robert L. Shoeman ${ }^{1}$, Ilme Schlichting ${ }^{\text {a,l }}$, Joachim Ullrich ${ }^{\text {a,i }}$

${ }^{a}$ Max Planck Advanced Study Group, Center for Free Electron Laser Science (CFEL), Notkestr. 85, D-22607 Hamburg, Germany

${ }^{\mathrm{b}}$ PNSensor GmbH, Römerstraße 28, D-80803 München, Germany

' Max-Planck-Institut für extraterrestrische Physik, Giessenbachstraße, D-85741 Garching, Germany

${ }^{\mathrm{d}}$ Max-Planck-Institut für Physik, Föhringer Ring 6, D-80805 München, Germany

e MPI Halbleiterlabor, Otto-Hahn-Ring 6, D-81739 München, Germany

${ }^{\mathrm{f}}$ Technische Universität Berlin, Institut für Optik und Atomare Physik, Hardenbergstraße 36, D-10623 Berlin, Germany

${ }^{g}$ DESY, Notkestr.85, D-22607 Hamburg, Germany

${ }^{\text {h }}$ University of Siegen, Emmy-Noether Campus, Walter Flex Str. 3, D-57068 Siegen, Germany

${ }^{i}$ Max-Planck-Institut für Kernphysik, Saupfercheckweg 1, D-69117 Heidelberg, Germany

${ }^{\mathrm{k}}$ Weierstrass Institut, Berlin, Mohrenstr. 39, D-10117 Berlin, Germany

${ }^{1}$ Max-Planck-Institut für medizinische Forschung, Jahnstr. 29, D-69120 Heidelberg, Germany

${ }^{\mathrm{m}}$ Max-Planck-Institut für biophysikalische Chemie, Am Fassberg 11, 37077 Göttingen, Germany

\section{A R T I C L E I N F O}

\section{Article history:}

Received 21 July 2009

Received in revised form 28 October 2009

Accepted 18 December 2009

Available online 4 January 2010

Keywords:

PnCCD

Parallel readout

Full depletion

Back illuminated

Radiation hard

Visible light

UV light and X-ray detection

CAMP chamber

Reaction microscope

Velocity map imaging

$\mathrm{X}$-ray imaging

X-ray spectroscopy

Free electron laser

\begin{abstract}
A B S T R A C T
Fourth generation accelerator-based light sources, such as VUV and X-ray Free Electron Lasers (FEL), deliver ultra-brilliant $\left(\sim 10^{12}-10^{13}\right.$ photons per bunch) coherent radiation in femtosecond $(\sim 10-$ $100 \mathrm{fs}$ ) pulses and, thus, require novel focal plane instrumentation in order to fully exploit their unique capabilities. As an additional challenge for detection devices, existing (FLASH, Hamburg) and future FELs (LCLS, Menlo Park; SCSS, Hyogo and the European XFEL, Hamburg) cover a broad range of photon energies from the EUV to the X-ray regime with significantly different bandwidths and pulse structures reaching up to $\mathrm{MHz}$ micro-bunch repetition rates. Moreover, hundreds up to trillions of fragment particles, ions, electrons or scattered photons can emerge when a single light flash impinges on matter with intensities up to $10^{22} \mathrm{~W} / \mathrm{cm}^{2}$.

In order to meet these challenges, the Max Planck Advanced Study Group (ASG) within the Center for Free Electron Laser Science (CFEL) has designed the CFEL-ASG MultiPurpose (CAMP) chamber. It is equipped with specially developed photon and charged particle detection devices dedicated to cover large solid-angles. A variety of different targets are supported, such as atomic, (aligned) molecular and cluster jets, particle injectors for bio-samples or fixed target arrangements. CAMP houses $4 \pi$ solid-angle ion and electron momentum imaging spectrometers ("reaction microscope", REMI, or "velocity map imaging", VMI) in a unique combination with novel, large-area, broadband ( $50 \mathrm{eV}-25 \mathrm{keV})$, highdynamic-range, single-photon-counting and imaging X-ray detectors based on the pnCCDs.

This instrumentation allows a new class of coherent diffraction experiments in which both electron and ion emission from the target may be simultaneously monitored. This permits the investigation of
\end{abstract}

\footnotetext{
* Corresponding author at: Max-Planck-Institut für extraterrestrische Physik, Giessenbachstraße, D-85741 Garching, Germany.

E-mail addresses: nik@hll.mpg.de, nils.kimmel@hll.mpg.de (N. Kimmel).
} 
dynamic processes in this new regime of ultra-intense, high-energy radiation-matter interaction. After an introduction into the salient features of the CAMP chamber and the properties of the redesigned REMI/VMI spectrometers, the new $1024 \times 1024$ pixel format pnCCD imaging detector system will be described in detail. Results of tests of four smaller format $(256 \times 512)$ devices of identical performance, conducted at FLASH and BESSY, will be presented and the concept as well as the anticipated properties of the full, large-scale system will be elucidated. The data obtained at both radiation sources illustrate the unprecedented performance of the X-ray detectors, which have a voxel size of $75 \times 75 \times 450 \mu \mathrm{m}^{3}$ and a typical read-out noise of 2.5 electrons (rms) at an operating temperature of $-50{ }^{\circ} \mathrm{C}$.

(c) 2010 Elsevier B.V. All rights reserved.

\section{Introduction}

Since the beginning of this decade, several laboratories worldwide have decided to build a new generation of extremely intense, coherent and short-pulsed VUV and X-ray light sources: the (X-ray) free electron lasers or (X-)FELs. These revolutionary machines deliver up to $10^{13}$ photons in $10-100 \mathrm{fs}$ bursts, in small, parallel beams with an energy uncertainty of $\Delta E / E \sim 10^{-3}$. They thus reach peak brilliances up to nine orders of magnitude higher than those achieved at the most advanced third-generation synchrotrons. Due to the small source size, the beam can be focused down to submicron spots, giving light intensities in the focus of up to $10^{22} \mathrm{~W} / \mathrm{cm}^{2}$. The first machine of this kind was the Free electron LASer at Hamburg, FLASH at DESY [1]. FLASH has been operational since 2005 and produces light at wavelengths between 6 and $40 \mathrm{~nm}$ (i.e., $30 \mathrm{up}$ to $200 \mathrm{eV}$ ) and pulse durations of about $25 \mathrm{fs}$. The next large facility to come online and presently in its commissioning phase is the Linac Coherent Light Source (LCLS) at SLAC in Menlo Park. LCLS is designed to deliver X-rays from below 0.8 up to $8 \mathrm{keV}$ at $100 \mathrm{fs}$ pulse duration [2]. While their basic concepts are identical, the practical realization and the operational schemes of FLASH and LCLS are quite different. FLASH employs super-conducting accelerator cavities with a fundamental repetition rate of 5 or $10 \mathrm{~Hz}$ and can optionally deliver up to 3000 (design value) micro-bunches per macro-pulse train, while LCLS will operate with conventional accelerator cavities at a constant repetition rate between 30 and $120 \mathrm{~Hz}$. In 2010, the SPring8 Compact SASE Source (SCSS) will be commissioned in Japan [3] with beam parameters similar to LCLS and a repetition rate of $60 \mathrm{~Hz}$. Finally, in 2014, another new facility will be opened to the scientific public, the X-ray Free Electron Laser, the European XFEL, in Hamburg [4]. The European XFEL will have a photon bunch structure similar to the one at FLASH. Every $100 \mathrm{~ms}, 3000$ photon pulses, equally spaced in time by $200 \mathrm{~ns}$, will be generated. After $600 \mu \mathrm{s}$, there will be a pause of $99.4 \mathrm{~ms}$, which is the cooling phase of the cryogenic super-conducting accelerator cavities. This means that on average 30,000 X-ray pulses will be delivered per second, providing 250 times the mean luminosity of LCLS and about 300 times the total photon flux achieved at PETRA III at DESY, the most advanced synchrotron in this energy range.

Accordingly, it is envisioned that major breakthroughs in different scientific disciplines ranging from physics and chemistry to material and life sciences will be achieved with these discovery machines. Foremost among them is the dream to follow the "making and breaking" of molecular bonds in real-time, i.e., to record a "molecular movie". In biology, one hopes to obtain structural information with sub-nanometer resolution of materials beyond the scope of conventional crystallographic approaches. These include nano- or non-crystalline proteins, macromolecular assemblies, viruses and cells. If successful, this approach would allow another access to the structure of membrane proteins which constitute about $60 \%$ of all therapeutic drug targets and are notoriously difficult to crystallize. In general, due to their high coherence, brilliance and short pulse time properties, FELs allow structural information to be obtained as a function of time, which will allow research in the femtosecond time regime. Beyond applications in biology and chemistry, this research is expected to be of utmost importance for the understanding of strongly correlated or magnetic materials, of phase transitions in liquids and solids, of melting processes at surfaces or in the bulk, of electron dynamics in atoms and molecules and of strongly correlated plasmas.

While these new machines promise unprecedented scientific possibilities, their properties also pose extreme challenges to the experimental instrumentation. For example, in some cases, scattered photon images have to be recorded at up to $5 \mathrm{MHz}$ frame readout rates or, in other cases, hundreds, thousands or even more electrons and ions have to be detected per shot. Beyond the requirements with respect to the performance of the detectors themselves, petabytes of data are recorded and have to be analyzed within reasonable times. Moreover, fundamental scientific questions arise beyond the "mere" technological challenges when penetrating this true terra incognita of light-matter interaction, which is orders of magnitude away from our present experience in energy density at the given photon energies. Thus, one of the decisive open questions regarding coherent imaging of biomolecules in the gas phase is whether the objects can be imaged before they are destroyed in the super-intense light flash. Since each single shot image will be a 2 dimensional projection of a 3 dimensional object, it will be necessary to know or to calculate the relative orientation of the molecule for each single shot before calculating a 3 dimensional reconstruction.

In order to meet some of these challenges concerning the instrumentation and to explore the relevant many-particle dynamics in this uncharted territory, we have designed the CFEL-ASG Multipurpose (CAMP) chamber. Proposed at the end of 2007 and designed in 2008 after extensive discussions within the scientific community, this chamber was built, tested and operated in 2009. The CAMP chamber houses the world's largest pnCCD chips. These detectors operate with a frame readout rate of up to $200 \mathrm{~Hz}$ and are thus fully capable of meeting the requirements at LCLS (up to $120 \mathrm{~Hz}$ ) and SCSS (up to $60 \mathrm{~Hz}$ ), as well as the $10 \mathrm{~Hz}$ macro-bunch operation scheme at FLASH or the European XFEL. The high-speed European XFEL and FLASH operation at up to $5 \mathrm{MHz}$ is expected to be coped with the recently proposed DePFET active pixel sensors and hybrid pixel detector arrays [5]. In addition, large-solid-angle momentum imaging spectrometers for emitted electrons and ions, which are commonly referred to as "reaction microscopes" (REMI) [6] or "velocity map imaging" (VMI) systems [7], have been redesigned and upgraded in order to enable simultaneous operation with the pnCCDs and to accommodate the fact that hundreds or even thousands of electrons and ions are emitted per shot. This combination of pnCCD photon detection with advanced charged-particle detection is expected to deliver detailed information on the interaction dynamics of intense VUV and X-ray FEL pulses with matter, to help uncover and subsequently possibly modify the destruction mechanisms of bio-particles to be imaged, to find schemes to dynamically align molecules for different shots by inspecting their fragmentation and scattering patterns shot by shot, etc. 
Thus, we envision that the CAMP chamber will serve a broad community encompassing coherent X-ray scattering and lensless imaging in material science (CXI), single particle and biomolecule imaging (SPB), femtosecond diffraction imaging experiments (FDE), $\mathrm{X}$-ray photon correlation spectroscopy (XPC), high energy density matter (HED) research, atomic and molecular physics (AMO), science with small quantum systems (SQS), X-ray absorption spectroscopy (XAS) and many more research areas to come. The unique combination of cutting edge imaging detectors along with the multi-user capability of the design was impressively affirmed by the fact that about 30\% of all approved (first campaign until December 2009) and proposed (second campaign starting in March 2010) experiments at LCLS plan to make use of the CAMP chamber. Moreover, a partial copy of the CAMP setup is presently under development for the Japanese SCSS free electron laser and has been designed such that our pnCCD detector array including chambers C2 and C3 (see Fig. 1) can be directly implemented. Experiments in Japan are planned to be performed in 2010.

This paper describes the general concept of the CAMP chamber, the REMI/VMI setup and the X-ray imagers. Since the REMI and VMI concepts have been discussed previously [6], we will concentrate on the changes and improvements necessary to combine them, for the first time, with photon detection. We will then focus on the detailed description of the pnCCD detector concept and elucidate how the pnCCD will meet the performance requests put forward by the users of the CAMP chamber. We will discuss the physical limitations of the system in terms of readout speed, noise performance, quantum efficiency, dynamic range, charge handling capacity, the dynamics of the charge cloud expansion and long term stability. We will further report on relevant previous results based on experiments performed at the BESSY synchrotron and at the FLASH facility. In this experiments, pnCCDs with an identical geometry (voxel size: $75 \times 75 \times 450$ $\mu \mathrm{m}^{3}$ with a format of $256 \times 256 \times 2$ ) were tested at a wide range of energies $(30 \mathrm{eV}-35 \mathrm{keV})$ and intensities and provide a basis for extrapolation to the anticipated performance of the pnCCD detectors in the full CAMP chamber.

\section{The CFEL-ASG Multipurpose (CAMP) chamber}

As outlined before, the CAMP setup is designed to achieve two major goals:

(i) To obtain the most complete data sets possible by momentum-resolved, large-solid-angle correlated detection of a large number of the electrons, ions and scattered or fluorescent photons emitted in each shot, similarly to the data collection strategies employed in high-energy physics experiments. This shall be achieved for different situations in which the FEL beam hits atoms, molecules, solids or plasmas or when optical alignment as well as pump lasers are fed in to allow for pumpprobe experiments on aligned molecules.

(ii) To guarantee high flexibility in order to enable the execution of a large variety of experiments. The ion and electron spectrometers allow for (coincident) many-particle, ion and electron momentum spectroscopy, as well as for velocity map imaging in combination with various jet targets. These components can be readily removed and replaced by other devices, such as fixed target setups, particle injectors or highresolution electron time-of-flight (TOF) spectrometers. Moreover, as will be illustrated below, the photon imaging detectors can even be mounted on the side of the incoming beam for more efficient detection of back-reflected or fluorescence light.

\subsection{General concept, layout and dimensions}

Fig. 1 depicts the complete CAMP setup including the support and the Helmholtz coils for generating the magnetic projection field for the electrons in the REMI. The FEL beam enters the chamber from the left and can exit towards a beam dump to the right. The device consists of four sections, CO-C3. From left to right, it starts with a differential pumping stage $(\mathrm{CO})$, which a

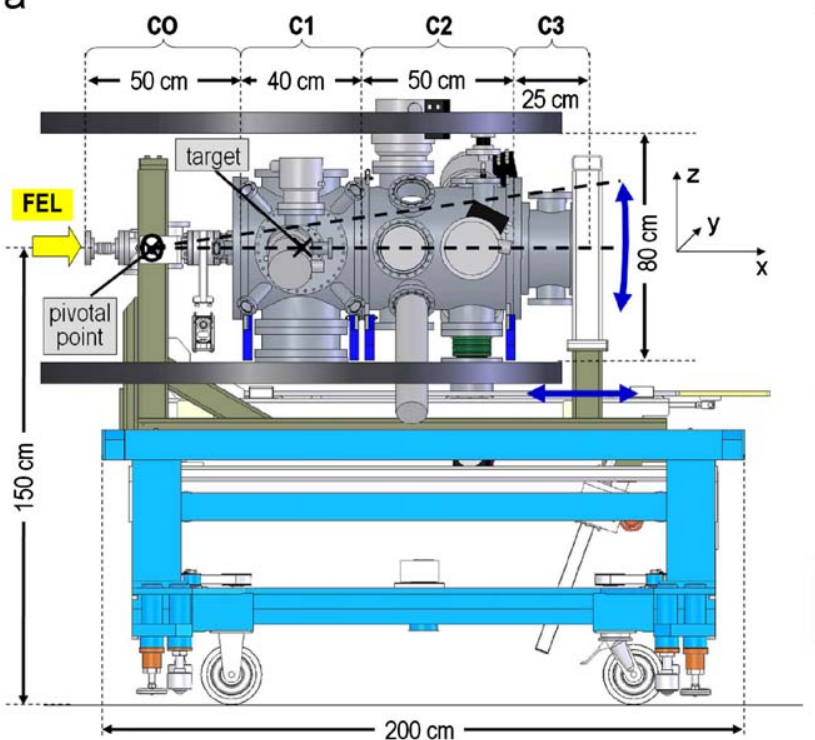

b

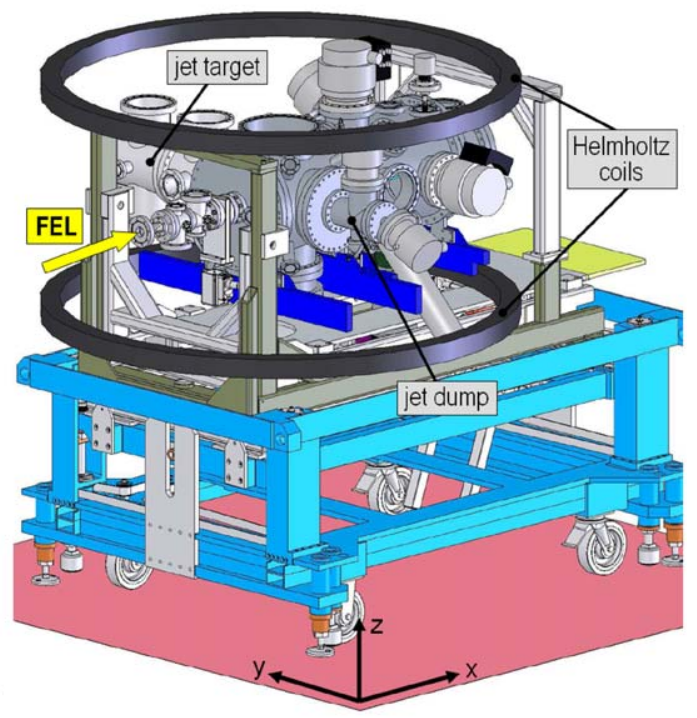

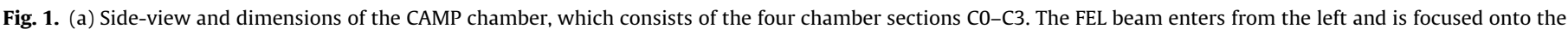

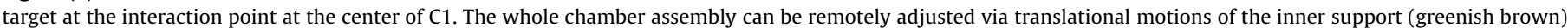

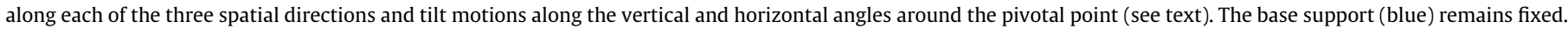

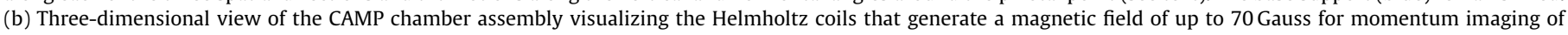

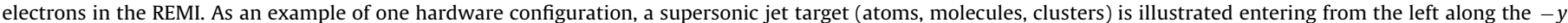
direction, with the jet dump on the opposite side. Large, removable flanges permit simple hardware exchanges. 
ensures that the beamline vacuum requirements are met even for main chamber pressures up to $10^{-6}$ mbar as may be present for injection of dense target beams. The first main chamber (C1), which has the FEL-sample interaction point at its center, houses the REMI or VMI spectrometers in the vertical direction (see Fig. 2a), and the jet-target, particle injectors or fixed-target setups. The first movable pnCCD detector array, pnCCD1, is located in the next large chamber (C2) whereas the second detector array, pnCCD2, is accommodated in C3 (see Fig. 2b). C1 and C2 have a $\varnothing 400 \mathrm{~mm}$ clear bore along the laser beam direction, whereas $\mathrm{C} 3$ has a $\varnothing 250 \mathrm{~mm}$ clear bore.

The design base pressure in the whole chamber is typically around $10^{-8}$ mbar, but can be lowered to $10^{-10} \mathrm{mbar}$ if required for particular experiments. To be able to reach this ultra-high vacuum range, flexible and easy-to-mount helicoflex gaskets are used for the $400 \mathrm{~mm}$ flanges. To allow for remote threedimensional position and angular alignment, which is crucial for the proper operation of the pnCCDs, the whole chamber assembly is mounted on an inner support (greenish in Fig. 1) which can be moved independently along the $x, y$ and $z$ directions by \pm 50 , \pm 50 and $\pm 150 \mathrm{~mm}$, respectively, with respect to the main support (blue in Fig. 1), which remains fixed. Thus, the target point can be adjusted exactly to the FEL beam. Thereafter, the center of pnCCD2, which is fixed within $\mathrm{C} 3$, can be aligned with respect to the FEL beam direction by rotating the whole assembly in the horizontal as well as vertical directions around a fixed pivotal point, each by an angle of about $\pm 6^{\circ}$ as depicted in Fig. 1 a. Since this pivotal point does not coincide with the target location for technical reasons, an iterative procedure with subsequent 3D and angular alignment actions may be necessary in some cases.

The unique and new feature provided by the fully equipped endstation is the possibility to simultaneously operate electron, ion and photon momentum imaging detectors as illustrated in cross sections through $\mathrm{C} 1$ in Fig. 2a, as well as through $\mathrm{C} 1-\mathrm{C} 3$ in Fig. 2b. Conically shaped electrodes around the interaction point allow for a free line of sight to the interaction point from all directions and generate homogeneous (REMI) or inhomogeneous (VMI) electric fields to extract ions and electrons into different directions towards two-dimensional position and time-resolved multi-channel detectors. In the standard configuration, photons are detected via two pnCCD detector arrays placed downstream of the interaction point as depicted in Fig. 2b. In this figure, the
REMI/VMI was removed for better illustration of the pnCCDs, and as an example, a supersonic jet source is shown in combination with a fed-in laser that allows aligning the target molecules. We would like to stress that the full REMI/VMI setup can be included in a way that is fully compatible with the full position maneuverability of the pnCCD system.

Two full sets of imaging X-ray detectors, each with ca. $80 \times 80 \mathrm{~mm}^{2}, 1024 \times 1024$ pixels active detection area (Figs. 2, 7 and 9) are incorporated into the CAMP chamber. Both detector systems consist of two halves with $1024 \times 512$ pixels. They are mounted on an electro-mechanical sub-module (see also Fig. 10). In total, four identical sub-modules will be installed in the CAMP chamber. The first detector set (pnCCD1) is moveable (Figs. 2 and 4) along the beam direction over $250 \mathrm{~mm}$ with the closest position being $50 \mathrm{~mm}$ behind the focal point at the center of $\mathrm{C} 1$. The two halves of the first detector can each be moved vertically (i.e. perpendicular to the beam) by $45 \mathrm{~mm}$ in order to cover larger scattering angles and can also overlap to shrink the diameter of the central hole if needed. The sketch in the right part of Fig. 2 illustrates possible motions of pnCCD1. The second set, pnCCD2, is housed in C3 and attached via a CF-DN250 flange at C2 in a fixed geometry with a minimal distance of $500 \mathrm{~mm}$ from the intersection point (standard position), but can be moved to any longer distance (by inserting adaptor tubes). Either one or both of the two detectors sets can also be used in back-scattering geometry by just feeding in the FEL beam in reversed, $-x$ direction or by mounting either pnCCD2 or pnCCD1 at the upstream side of $\mathrm{C} 1$ with the incoming photon beam traversing the detectors through the center hole of $\varnothing 2.2 \mathrm{~mm}$. This photon detector geometry is ideally suited to back-reflect low-energy photon beams via multilayer mirrors in order to achieve smallest focus spots and, thus, highest intensities as recently demonstrated at FLASH (see e.g. [8]). We would like to further point out that mounting additional detector sets in the upstream direction is one natural, upcoming extension of CAMP in the future (Figs. 3-10).

\subsection{The target chamber and the reaction microscope setup}

The REMI/VMI spectrometer (outer $\varnothing 90 \mathrm{~mm}$ for VMI and $135 \mathrm{~mm}$ for REMI, shown in yellow in left part of Figs. 2), is mounted vertically in the standard setup such that it does not

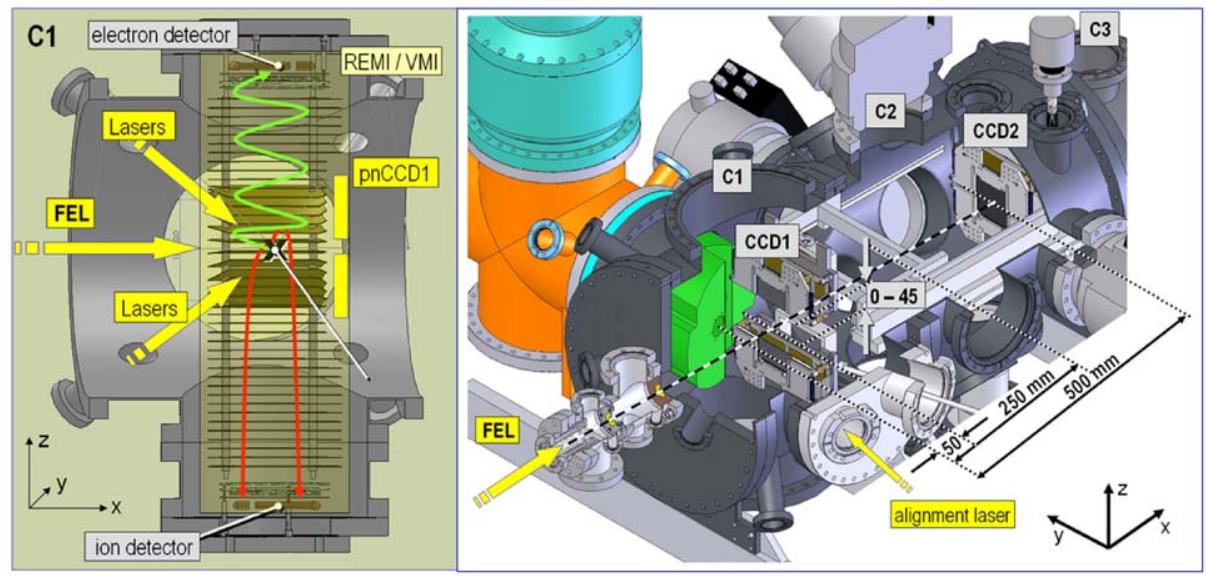

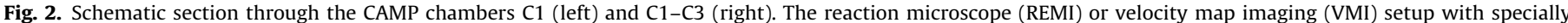

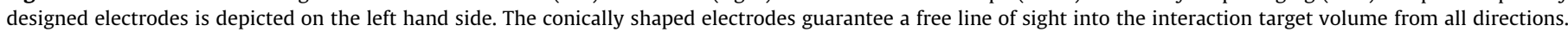

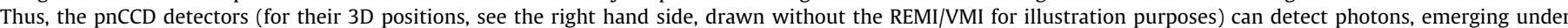

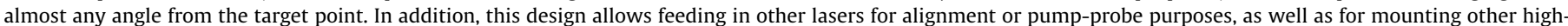

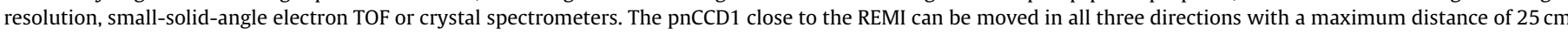

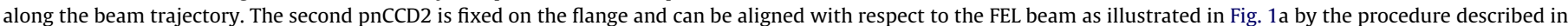
the text. 


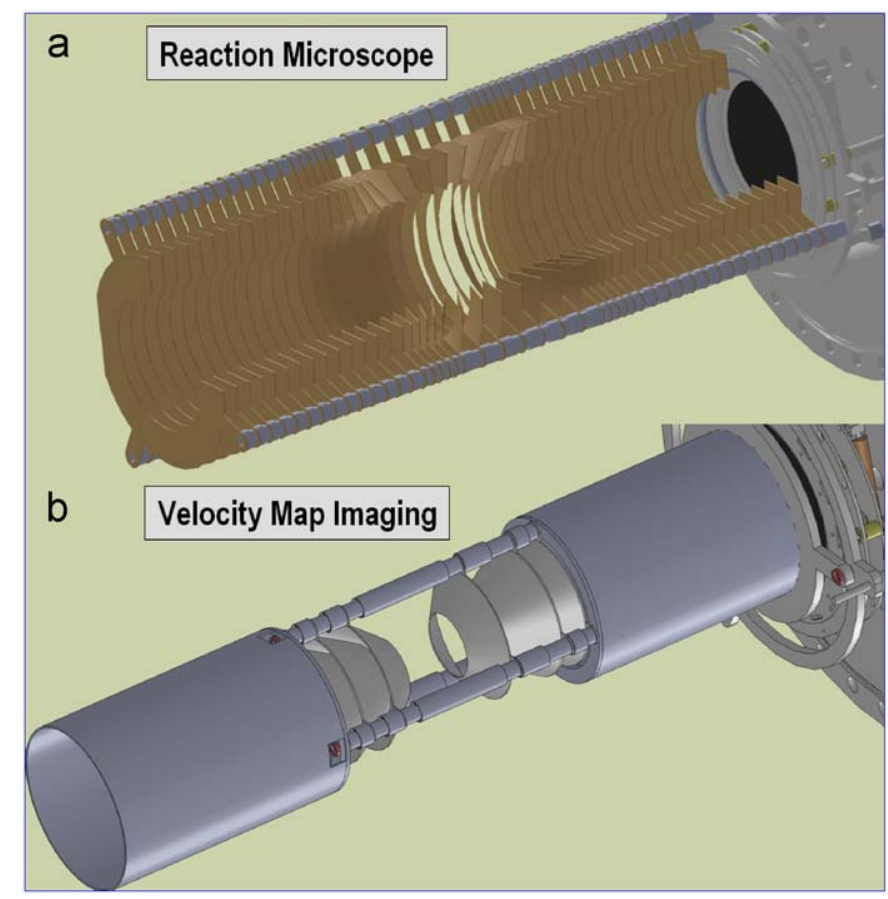

Fig. 3. $3 \mathrm{D}$ model of the CAMP reaction microscope (a) and the velocity map imaging (b) spectrometers. The total length of both devices is $400 \mathrm{~mm}$, and both are designed for $\varnothing 80 \mathrm{~mm}$ detectors. The conically shaped central electrodes are clearly visible. They provide a free line of sight for the pnCCDs over their complete active area and, at the same time, suppress stray light, which is, especially effective for the REMI geometry. For the REMI typical voltages are a few $\mathrm{V} / \mathrm{cm}$. The VMI is operated at $\mathrm{kV} / \mathrm{cm}$ for full solid-angle imaging of electrons up to $200 \mathrm{eV}$. With a magnetic field of up to 70 Gauss for the REMI, electrons emitted with energies of up to $2 \mathrm{keV}$ into the most unfavourable direction, transverse to the electric extraction field, are confined to a spiral trajectory with a diameter of about $70 \mathrm{~mm}$ and, thus, are detected with a solid angle of $50 \%$ of the full $4 \pi$ steradiant. Multichannel plate detectors are mounted at both ends and can be equipped with delayline anodes or phosphor screen CCD camera readout (see text).

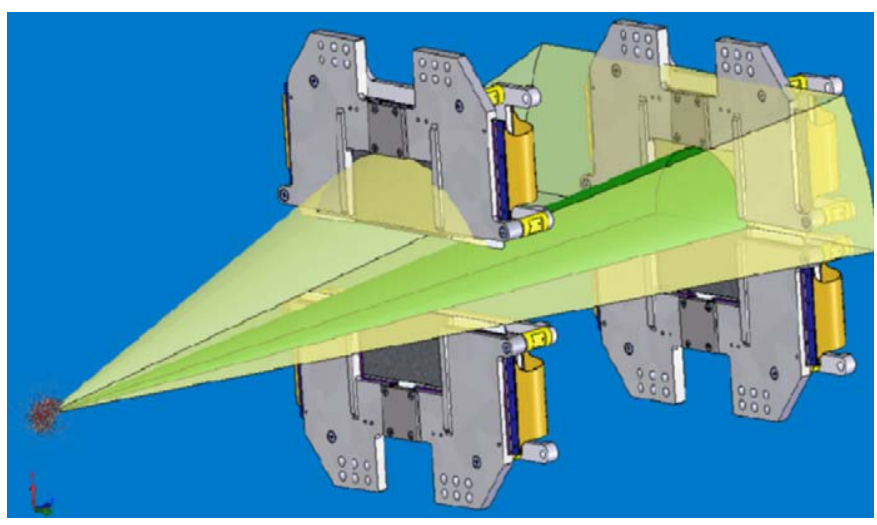

Fig. 4. Artist's view of the two pnCCD mountings. The two halves of the pnCCD on the left side (closest to the interaction point), CCD1, can be opened vertically by up to $90,45 \mathrm{~mm}$ each. Photons scattered under large angles can hence be detected, while the second (fixed) pnCCD2 records the X-rays under smaller angles. The distance between the detectors can be changed by moving the CCD1 system up to $250 \mathrm{~mm}$ along the beam axis. Moreover, if needed, CCD2 can be placed at any distance larger than $550 \mathrm{~mm}$ away from the interaction zone by simply introducing fixed spacer tubes of the desired length.

limit the motion of pnCCD1. This permits the mounting of different target jet devices along with lasers for alignment or pump-probe purposes. The middle part of each spectrometer consists of conically shaped, gold-plated aluminum electrodes that generate the homogeneous projecting (REMI, Fig. 3a) or inhomogeneous imaging (VMI, Fig. 3b) electric fields. Further outside, two solid drift tubes (VMI) or equally spaced potential rings (REMI) guarantee a field-free drift region. The conical shape of the central electrodes within the solid angle covered by the pnCCD detectors allows unrestricted view into the scattering volume. At the same time, the cones excellently suppress scattered stray radiation emerging from outside the interaction center.

Since the operation principles of REMI/VMI spectrometers have been described extensively in the literature, only the salient features are briefly outlined in the present contribution. For the REMI configuration, illustrated in Figs. 2a and 3a, the electric field forces all ions, independent of their initial momentum, onto parabolic trajectories (red lines in Fig. 2a) and projects them onto the detector. The electric field, usually a few $\mathrm{V} / \mathrm{cm}$, can in most cases be adjusted such that all ions of interest are registered. Measuring the time-of-flight (TOF) and the hit position, the trajectory and, thus, the full three dimensional momentum vector of each individual ion can be reconstructed. Electrons are pushed towards the other hemisphere by the electric field but cannot be efficiently projected onto the detector with moderate fields. Even though their momenta are typically very similar to those of the ions, the electron energies are several thousand times larger, and accordingly, keV projection voltages would be required, leading to times-of flight of a few nanoseconds. This is too short to achieve reasonable momentum resolution along the extraction field direction. In a REMI, the dilemma is solved by maintaining a small electric field but confining the electrons' transverse motion via an additional homogeneous (Helmholtz configuration, coil $\varnothing 160 \mathrm{~cm}$ ) magnetic field (here up to 70 Gauss), oriented parallel to the electric field and forcing them onto spiral trajectories as sketched in Fig. 2a (green line). Again, by measuring the TOF and the hit position, the full initial momentum vector can be retrieved (for details, such as the attainable resolution, see [6]). Both detectors are equipped with either square or hexagonal delay line anodes (Roentdek HEX80 [9]) read out by an eight-channel, $2 \mathrm{GHz}$ digitizer (Acquiris DC $82 \times 2$ ) recording the complete wave form of the signal. It has been demonstrated recently at the SCSS test facility that up to 150 ions emerging from the interaction of a single EUV-FEL pulse with a single cluster could be analyzed, with their individual 3D momentum vectors [10] determined independently. In principle, the REMI allows for the coincident detection of momentumresolved ions and electrons emerging from the same reaction. This feature has been used to explore sequential two-photon double ionization of $\mathrm{Ne}$ atoms in a kinematically complete experiment where two electrons and the ion have been detected in a triple coincidence [11]. Meanwhile, specially adapted REMIs have been successfully operated in many experiments at FLASH and the SCSS test facility [12-14] and the present design builds on this previous experience.

Alternatively, if even more fragments are ejected per FEL shot, the delay line anode can be replaced by a phosphor screen combined with a CCD camera readout, thus recording the image of electrons and/or ions without the magnetic field and applying non-homogeneous imaging voltages generated by the potential cones depicted in Fig. 3b. In this "velocity map imaging" [7] mode, only two-dimensional (position) images are generated and the time information is usually lost due to short flight times of the electrons resulting from the large imaging electric fields of up to $\mathrm{keV} / \mathrm{cm}$. Still, under certain conditions and/or for low-energy electrons, three-dimensional images can be retrieved (for details see [15]). Again, the applicability of this method at FELs has been proven recently at FLASH [16]. Since the standard VMI electrode configuration [7] had to be modified for our application, additional lenses are used to provide better space focusing [17]. 


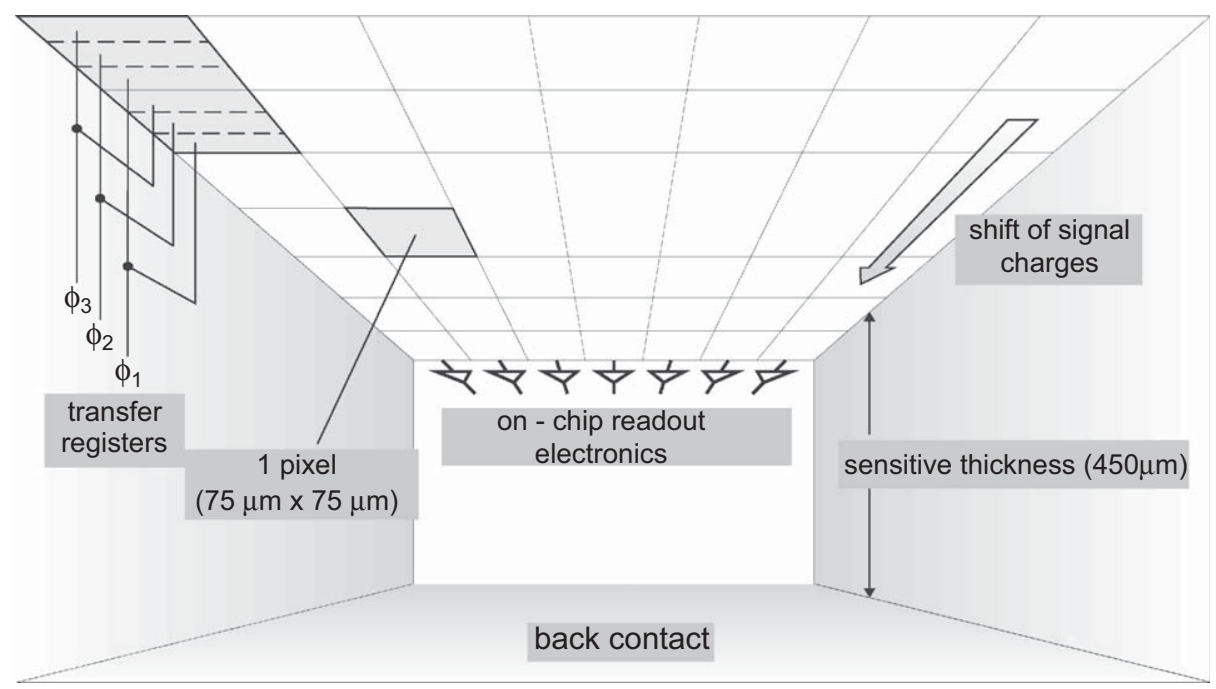

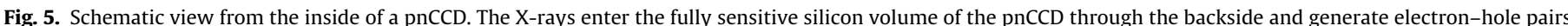

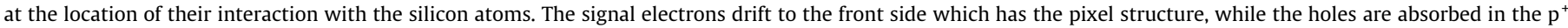

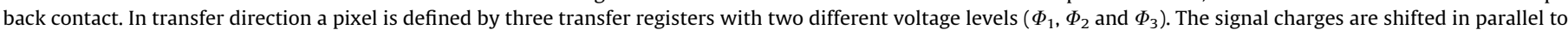

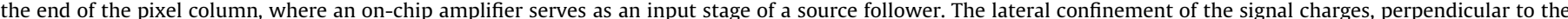

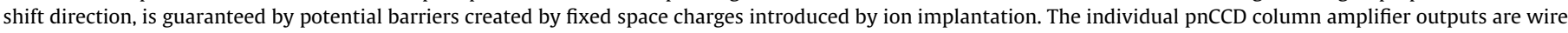
bonded in groups of 128 to an ASIC amplifier called CAMEX for further signal processing.

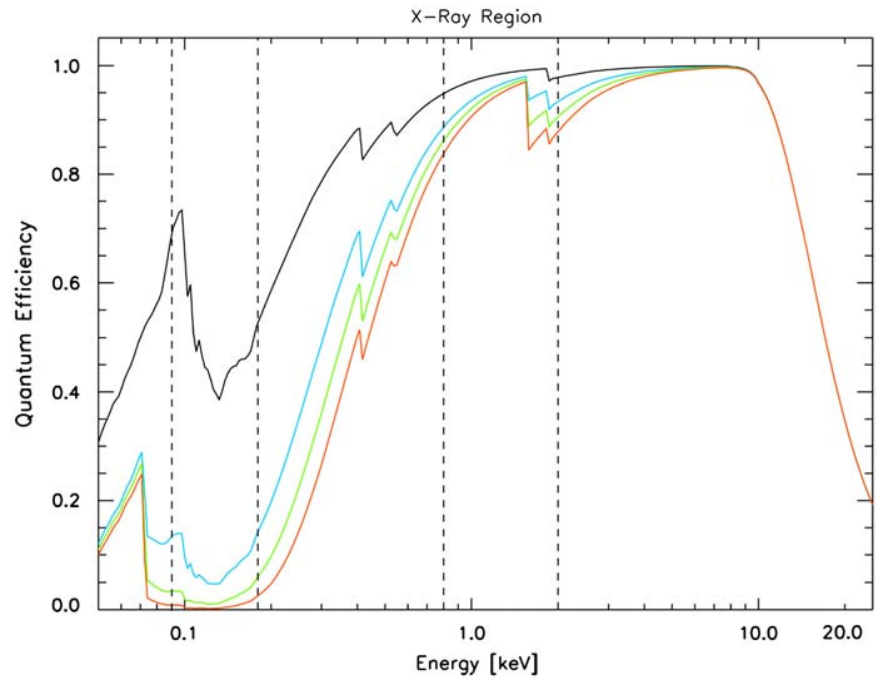

Fig. 6. Quantum efficiency of the pnCCDs as a function of energy for a $450 \mu \mathrm{m}$ thick pnCCD detector with different light blocking filters. The red curve shows the $\mathrm{QE}$ for an $\mathrm{Al}$ coating of $150 \mathrm{~nm}$, which has a light attenuation of more than $10^{6}$ in the visible region. The green curve corresponds to a $100 \mathrm{~nm}$ coating, the blue one to $50 \mathrm{~nm}$ of aluminum. The black curve denotes a configuration with only a thin $\mathrm{SiO}_{2}$ and $\mathrm{Si}_{3} \mathrm{~N}_{4}$ passivation layer. These layers are transparent for visible light. For FLASH operation at 90 and $180 \mathrm{eV}$, the QE is $15 \%$ with the $50 \mathrm{~nm}$ coating, while it is about $90 \%$ in the LCLS energy range. At energies up to $10 \mathrm{keV}$, the QE is very close to $100 \%$.

In the standard REMI/VMI operation mode, two opposing CF-DN250 flanges in the horizontal direction in C1, perpendicular to the FEL beam, allow for installation of various targets, such as various supersonic jets (see Figs. $1 \mathrm{~b}$ and $2 \mathrm{~b}$ ), particle injectors, cluster or water droplet sources and their appropriate jet dumps. In this fully equipped setup, C1, C2 and C3 are pumped via Turbo Molecular Pumps (TMPs) mounted on four of the eight CF-DN150 flanges surrounding $\mathrm{C} 2$. By this means, utmost flexibility is provided for using the ports of $\mathrm{C} 1$ exclusively for experimental and user needs. C1 was kept as short as possible along the beam direction $(400 \mathrm{~mm})$ because of the relatively short X-ray focus

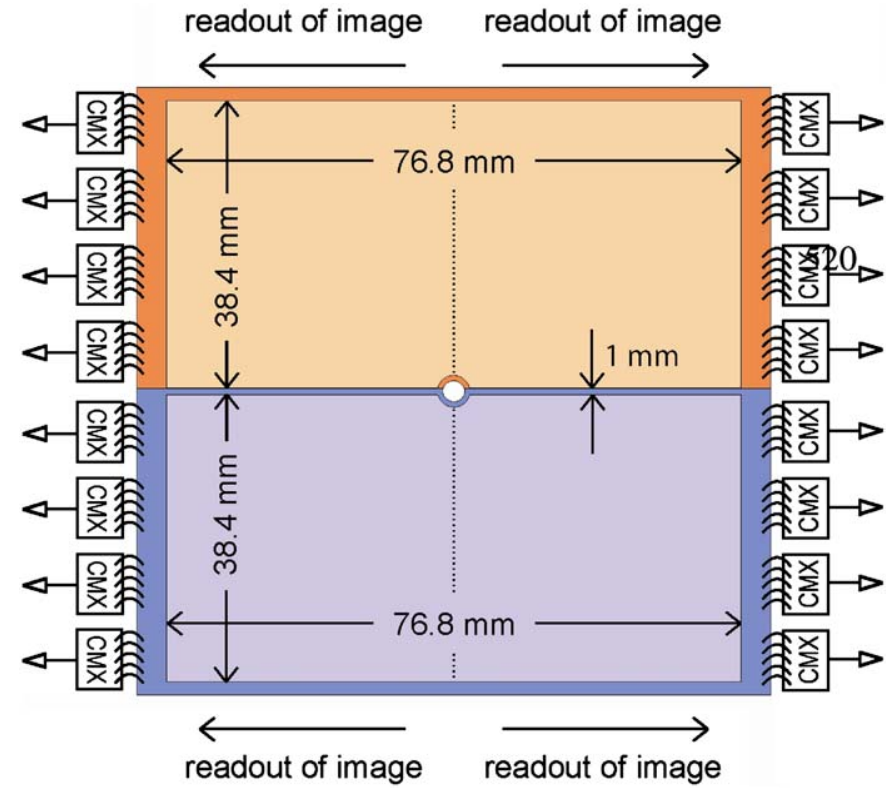

Fig. 7. Schematic layout of the two halves of the $1024 \times 1024$ pnCCD system. Each half has a format of $512 \times 1024$ pixels of $75 \mu \mathrm{m} \times 75 \mu \mathrm{m}$ size. The devices will be operated in a split frame mode, e.g. the upper (orange) pnCCD transfers the signal charges to the right $(512 \times 512)$ and to the left with the indicated device divider in the center. There is no insensitive gap between the two $512 \times 512$ units. Both halves $(1024 \times 512)$ are operated independently. The on-chip amplifiers feed their signals in the CAMEX signal processors. Preamplification, bandwidth limitation filtering, sample and hold multiplexing and line driving is performed in the CAMEX chips. The CAMEX outputs are directly received by 16 ADCs located in the vicinity of the warm side of the DAQ electronics. For interpretation of the references to colour in this figure legend, the reader is referred to the web version of this article.

lengths at some beamlines. Finally, the REMI/VMI setup is readily removable and other types of spectrometers (Thompson parabola, high-resolution electron TOF, magnetic bottle, high-resolution crystal or grating spectrometers, etc.) or fixed-target setups can be implemented instead. Supports in the chamber C1 allow mounting of apertures and fixed-target arrangements on 


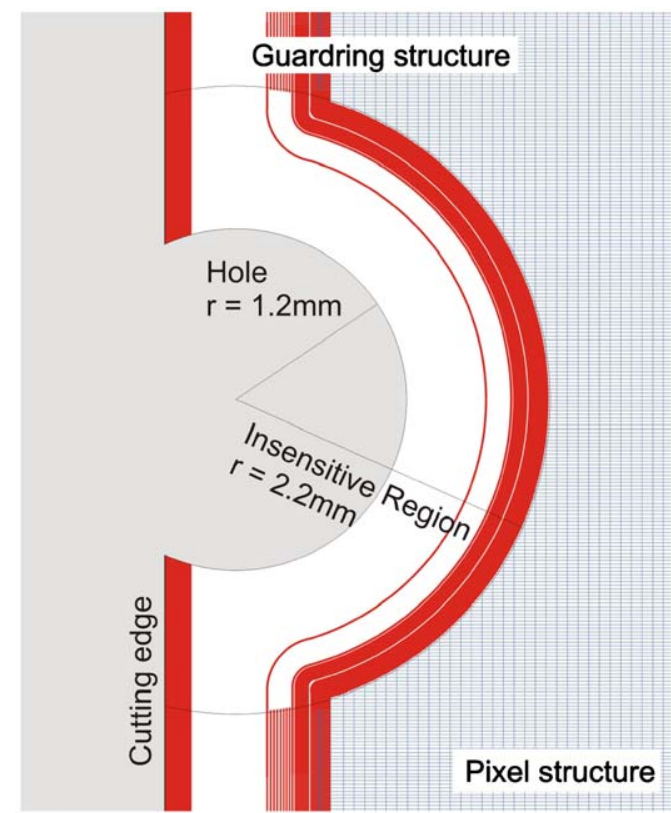

Fig. 8. Geometry around the center hole cut into the wafer with a special laser. The clear bore has a radius of $1.2 \mathrm{~mm}$. In the conservative case, another $1 \mathrm{~mm}$ is needed from the cutting edge to the first sensitive pixel. That area is partially filled with dedicated guard structures to reduce the electric field from the negatively biased backside towards the cutting edge. On the front side with the pixel structures, the breakdown problems are more relaxed. Later studies will try to reduce the $1 \mathrm{~mm}$ "safety area" to approximately $500 \mu \mathrm{m}$.

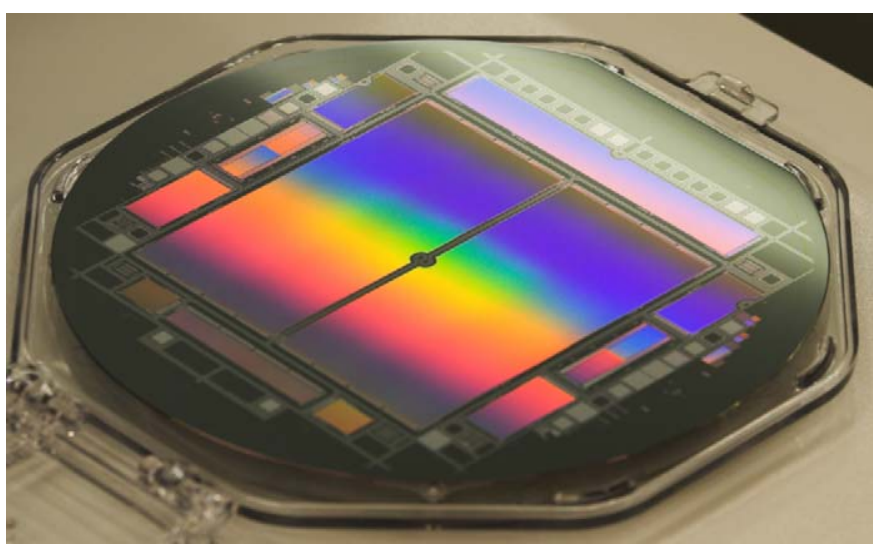

Fig. 9. Photograph of a $150 \mathrm{~mm}$ wafer with two pnCCDs and a couple of test devices. The hole in the center has a clear opening of $2.4 \mathrm{~mm}$, surrounded by $1 \mathrm{~mm}$ of insensitive area. Each detector half has a sensitive area of approximately $30 \mathrm{~cm}^{2}$ filled with $1024 \times 512$ pixels of $75 \times 75 \mu \mathrm{m}^{2}$. Laser cutting separates the chips to configure them for final mounting.

breadboards. Various CF-DN38 ports allow the coupling of optical lasers from different directions, the mounting of various highresolution VUV, X-ray or TOF electron spectrometers, beam monitors and much more. The $\varnothing 400 \mathrm{~mm}$ end-flange upstream contains additional ports for telescopes, laser injection, etc.

Thin optical and mechanical blocking filters can be inserted in front of the detector pnCCD1 to prevent optical light and lowenergy electrons from hitting the radiation sensitive windows of the X-ray imagers in the $\mathrm{C} 2$ and $\mathrm{C} 3$ chambers and to avoid chemical and mechanical contamination. The REMI magnetic field has the important side effect that it can effectively prevent electrons of up to $10 \mathrm{keV}$ from hitting the pnCCDs, if required. Depending on the FEL beam energy and the target, the number of

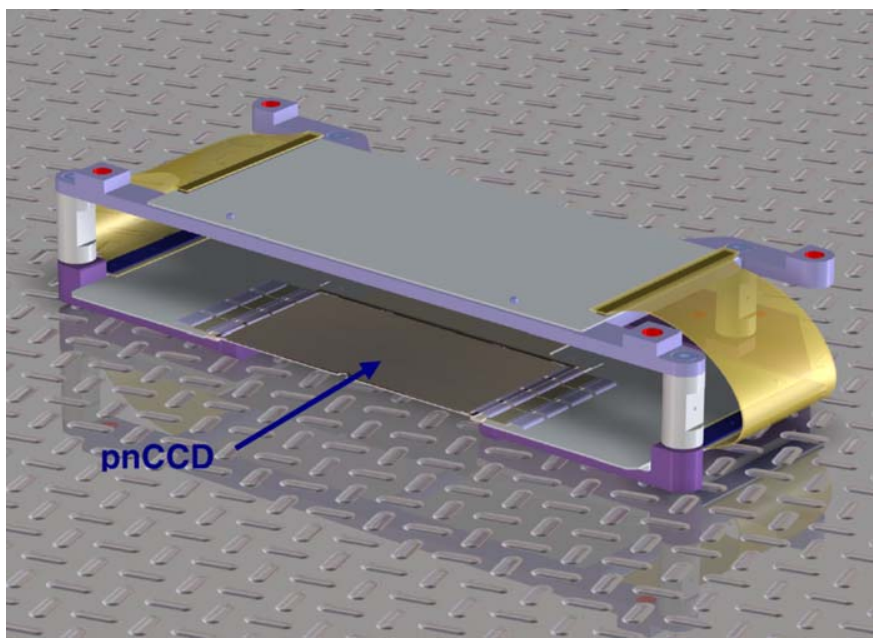

Fig. 10. Mechanical study of one of the movable halves of the detector system (sub-module). The pnCCD is the grey part in the center of the bottom layer, the CAMEX chips can be seen at the sides. They are wire bonded column-wise to the CAMEX chips located on the lower ceramic board. As the pnCCD is backilluminated, radiation will hit the detector from the bottom. The upper board contains filter components for the DC power supplies as well as control logic and line drivers. The lower part containing the CCD and CAMEX chips is kept at a temperature between $-50{ }^{\circ} \mathrm{C}$ and room temperature.

high-energy electrons produced might exceed that of scattered photons by orders of magnitude and therefore, could obscure many coherent imaging experiments. If desired, however, the magnetic field can be switched off and high-energy electrons can be detected as well with the pnCCDs. Here, we would like to emphasize that the pnCCDs are also suitable for angle-resolved and energy-dispersive detection of high-energy electrons from at least one up to hundreds of keV. Due to the extreme granularity of the detector's sensitive area, millions of electrons emitted per shot can be recorded simultaneously, a fact that has not yet been realized by the broad scientific community. Electron detection can be of decisive advantage for many experiments, such as the investigation of plasmas generated either by optical or the X-ray free electron laser pulses. Presently, the detection characteristics as a function of the electron energy of the pnCCDs is being investigated in a test setup and the results will be reported on in a separate, subsequent publication.

\section{3. pnCCDs for $\mathrm{X}$-ray imaging spectroscopy}

The concept of the CAMP pn-junction charge coupled device is based on the pnCCDs [18] developed for the X-ray satellite mission XMM-Newton [19]. XMM was launched in December 1999. Since then, the European Photon Imaging Camera with its pnCCD focal plane (EPICpn) has been delivering spectroscopic $\mathrm{X}$-ray images of unprecedented quality. It is working reliably and stably, without the need for changing the operation conditions compared to the ground calibrations. The maximum energy uncertainty at $6 \mathrm{keV}$ has been only $2 \mathrm{eV}$ over the past 10 years.

In contrast to the well-known, more conventional metaloxide-silicon based MOS CCDs, the pnCCDs are based on highresistivity n-type silicon (typ. $5000 \Omega \mathrm{cm}$ ) and a manufacturing process that makes use of both wafer surfaces for the sensor and electronics circuitry. This enables the full depletion of the entire silicon volume and, thus, makes use of the full depth as sensitive volume for the conversion of the incident X-rays into electronhole pairs. Since all components of the sensor are built up with 
pn-junctions instead of MOS structures, the device is denoted pnCCD.

A two-phase development program was started in spring 2007 in the framework of the CFEL-ASG to make specially designed pnCCD systems available to facilities where CFEL is scientifically involved. Phase I comprises the commissioning of a high-speed $1024 \times 1024$ focal plane module in 2009 and phase II aims at providing a four times larger format module of $2048 \times 2048$ pixels to be operational in $2012 / 2013$.

\section{1. pnCCD concept}

The pnCCD is fabricated on double-sided, polished, n-type $150 \mathrm{~mm}$ silicon wafers, typically $300-500 \mu \mathrm{m}$ thick. The resistivity $\rho$ of the bulk material varies between 3000 and $6000 \Omega \mathrm{cm}$ to allow full depletion at bias voltages far below electrical breakdown. Full depletion is achieved by reverse-biased voltages applied to pn-junctions on the front and backside of the CCD, i.e. to the transfer registers (located at the "front side" of the detector) and to the photon entrance window (called "back contact" or radiation entrance window, see Fig. 5). By the choice of appropriate voltages, a potential minimum for electrons is created in the n-type silicon with an absolute minimum at a depth of about $7 \mu \mathrm{m}$ from the front surface. Incoming X-rays enter the pnCCD from the backside through the large-area, thin $\mathrm{p}^{+}$implanted radiation entrance window. The energy needed to create an electron-hole pair in silicon is about $3.7 \mathrm{eV}$ at a detector temperature of around $-50{ }^{\circ} \mathrm{C}$. The electron-hole pairs, generated by the X-ray photons, are separated by the applied electric field of approximately $0.5 \mathrm{~V} / \mu \mathrm{m}$. The electrons drift to their potential minimum which is located inside an epitaxial layer of lower resistivity (approximately $40 \Omega \mathrm{cm}$ ) on top of the bulk material. During photon integration time, the signals are stored in the potential wells of the pixels that are built up by three transfer registers with two different voltage levels applied. On command, the signal electrons are transferred with high-speed from the location where they have been stored towards the readout node. As with earlier pnCCDs, each transfer channel is terminated by an anode coupled to an on-chip amplifier (see Fig. 5), thus avoiding the time consuming serialization (multiplexing) of a highimpedance signal. This concept was first proven with pnCCDs in 1990 in the test phase of the XMM mission [20].

The $\mathrm{n}^{+}$anode, i.e. the arrival node of the signal charges at the end of a column, is connected to the $\mathrm{p}^{+}$gate of an n-channel JFET, which is monolithically integrated on the pnCCD-chip (on-chip readout electronics in Fig. 5). Wire bonds connect the source of the JFET to a dedicated CAMEX preamplifier channel [21] in a source follower configuration (see Fig. 13).

The extended sensitive depth of $450 \mu \mathrm{m}$ enhances the quantum efficiency in the near infrared regime up to $1.1 \mu \mathrm{m}$ wavelength as well as the X-ray region to about $50 \%$ at $15 \mathrm{keV}$ (see Fig. 6). The focal plane is cooled to approximately $-50{ }^{\circ} \mathrm{C}$ to minimize variations in pnCCD properties (noise, gain and offset) and to improve long term stability of the devices.

\section{2. $p n C C D$ for free electron laser applications}

We have proposed pnCCD modules as 2-dimensional X-ray sensitive focal plane detectors for use at fourth generation $\mathrm{X}$-ray light sources. These imagers can measure either the X-ray intensity precisely or the X-ray energy in the case of single photons in one pixel. The relevant set of required performance parameters for applications at the new X-ray laser sources are listed in Table 1 . The main feature of the two-dimensional detector system is its capability to distinguish one photon from no
Table 1

Experimental requirements for the 2-D detectors and current pnCCD properties.

\begin{tabular}{|c|c|c|}
\hline Parameter & $\begin{array}{l}\text { 2-D imager requirements for } \\
\text { FLASH, LCLS, SCSS and XFEL } \\
\text { requested by the user community }\end{array}$ & $\begin{array}{l}\text { pnCCD } \\
\text { properties }\end{array}$ \\
\hline Single photon resolution & Yes & Yes \\
\hline Energy range & $0.05-24 \mathrm{keV}$ & $0.05-25 \mathrm{keV}$ \\
\hline Signal rate/pixel/bunch & $10^{3}\left(10^{5}\right)$ & $10^{3}$ at $2 \mathrm{keV}$ \\
\hline Charge handling capacity & - & $\begin{array}{l}\text { Approx. } \\
5 \times 10^{5} \\
\text { electrons per } \\
\text { pixel }\end{array}$ \\
\hline Quantum efficiency & $>0.8$ & $\begin{array}{l}>0.8 \text { from } \\
0.3-12 \mathrm{keV}\end{array}$ \\
\hline Number of pixels, format & $512 \times 512$ (min.) & $\begin{array}{l}1024 \times 1024 \\
\text { and } \\
2048 \times 2048\end{array}$ \\
\hline Pixel size & $<100 \times 100 \mu \mathrm{m}^{2}$ & $75 \times 75 \mu \mathrm{m}^{2}$ \\
\hline $\begin{array}{l}\text { Frame rate repetition rate } \\
\text { (except XFEL's } 5 \mathrm{MHz} \\
\text { operation) }\end{array}$ & $5-120 \mathrm{~Hz}$ & $\begin{array}{l}\text { Continuous up } \\
\text { to } 200 \mathrm{~Hz}\end{array}$ \\
\hline Externally triggerable & - & Yes \\
\hline Integrated center hole & $\emptyset 3 \mathrm{~mm}$ & $\varnothing 2.4 \mathrm{~mm}$ \\
\hline $\begin{array}{l}\text { EUROPEAN XFEL burst } \\
\text { mode }\end{array}$ & $5 \mathrm{MHz}$ (3000) bunches & Not applicable \\
\hline Readout noise & $<150$ electrons & $\begin{array}{l}20 \mathrm{e}^{-} \text {(low } \\
\text { gain), } 2 \mathrm{e}^{-} \\
\text {(high gain) }\end{array}$ \\
\hline
\end{tabular}

photon over the full energy range and to supply a sufficiently large dynamic intensity range at a superior frame read-out repetition rate of $200 \mathrm{~Hz}$. The measurement of up to one thousand $2 \mathrm{keV}$ photons per pixel must be achieved with an intensity resolution of better than $\pm \sqrt{N}$, with $N$ being the number of photons. Of course, every single shot has to be read out before the next 20-150 fs photon pulse hits the sample. In case of LCLS, this means that the frame time has to be shorter than $8 \mathrm{~ms}$. Energy resolution as such was originally not desired explicitly by the users. However, the possibility to separate the higher harmonics coming with the photon beam as well as the capability to measure fluorescence light and to discriminate against the elastically scattered photons was considered to be very helpful and essential for some of the anticipated experiments.

\subsection{Phase I: the $1024 \times 1024$ format}

After the successful test of the smaller format detectors (see Section 3.4), design and fabrication of the new focal plane imagers for FEL (synchrotron) experiments were started. As can be seen in Figs. 7 and 9, the $7.6 \times 7.6 \mathrm{~cm}^{2}$ large detector was split in two halves with a central hole (Fig. 8). Each single pnCCD chip has a format of $512 \times 1024$. This layout permits the placement of the two movable pnCCD detector halves around the X-ray beam to cover a larger angular range with the first system, CCD1 (see Fig. 4). Readout of the pnCCDs is performed in a split frame mode, i.e. every pnCCD half is transferred to the right or left (see Fig. 7). The front end electronics system will be equipped with 16 ADCs running at $10 \mathrm{MHz}$ each with a resolution of $14 \mathrm{bit}$. The control electronics (sequencer, ADCs, data pre-processor, data acquisition, power supplies) is a cPCI-based modular 19 in. compatible unit.

As usual for our systems, all pnCCD columns are shifted and processed in parallel. The CAMEX ASIC (see Fig. 13), receives the preamplified signals and then performs further amplification, gain selection, signal processing, sample and hold and multiplexing (typ. 128:1), and line driving. The output, i.e. 128 channels, is multiplexed after the signal processing with typically $10 \mathrm{MHz}$ per CAMEX output node to an ADC with 14-bit resolution. 
Our laboratory systems have either 2,4 or 8 output nodes. The digitized data are transferred to a computer through four optical $2.5 \mathrm{GHz}$ links.

The pnCCD data acquisition system can be upgraded to $n \times 4$ output nodes. Every 4-channel ADC unit is housed in a $19 \mathrm{in}$. rack. The pnCCD system runs on a LINUX-based platform. Data reduction can be performed with the help of an Field Programmable Gate Array (FPGA) located on the ADC motherboard. Gain correction, offset correction, common mode rejection, etc. are performed immediately after digitization. The final data analysis is done offline by the user, as the type of data analysis varies extensively for the different experiments. Data compression can be implemented in an FPGA directly behind the ADC.

The measurements shown in Section 3.5 have been performed with an identical pnCCD system except for the format being $2 \times 256^{2}$ instead of $1024^{2}$. In the LCLS test case, the two pnCCD systems produce $2 \times 10^{6}$ ( 2 cameras of $10^{6}$ pixels each) $\times 120$ (frames per second) $\times 2$ ( 2 byte amplitude resolution) byte per second, i.e. almost 0.5 GByte of data per second, which translates to 40 TByte per day.

\subsection{Phase ii: the $2048 \times 2048$ format}

Experimenters have requested larger fields of view and a smaller center hole, while retaining the flexibility of translating the detector halves. The ultimate reduction of the insensitive areas at the edges will be the aim of an upcoming technological study. The preliminary design and layout of a large-format subsystem $(1024 \times 1024$ as shown in Fig. 11a) is already in progress and we plan to start the production in 2010 after the first feedback from the Phase I system has been received. Fig. 11b shows the basic geometrical arrangement of the 4 Megapixel $\mathrm{X}$-ray camera. It is composed of 4 identical subsystems which are joined together as shown below. The full system will have a sensitive area of $236 \mathrm{~cm}^{2}$ with approximately $0.8 \%$ of insensitive area along the joints of the four detectors. The physical parameters of the Phase II system, such as noise, quantum efficiency, pixel size, charge handling capacity, energy range, etc., are identical to those of the Phase I system. Since the maximum number of transfers per CCD is 1024 instead of the 512 of the Phase I system, the readout of the Phase II pnCCD must be accelerated moderately to cope with the $120 \mathrm{~Hz}$ frame rate of LCLS.

Correspondingly, the readout and data acquisition electronics will be upgraded to cope with the data rate of 2 GByte per second. In total, $64 \mathrm{ADCs}$ will be needed to process the data in real-time. The processing time for one pixel has to be shorter than $7 \mu \mathrm{s}$. This translates to a CAMEX analog multiplexing (64:1) speed of approximately $10 \mathrm{MHz}$ which is already achieved with today's systems.

\subsection{Charge spread in silicon detectors}

X-rays below $30 \mathrm{keV}$ interacting with silicon deposit their energy predominantly through photoelectric absorption. Other processes like Compton scattering can be safely neglected. If the energy of the incident X-ray is above $1.83 \mathrm{keV}$, the most probable process is K-shell ionization. In this case, the excess energy above the K-ionization level is transferred as kinetic energy to the outgoing electron, which then interacts with electrons in the same or neighboring silicon atoms. As the primary silicon atom is excited due to the missing $\mathrm{K}$-shell electron, it relaxes either through the emission of a $1.74 \mathrm{keV}$ photon or via the ejection of an Auger electron of about $1.55 \mathrm{keV}$, both of which deposit their energies in close vicinity. The size of the charge cloud as a function of the incident energy was approximated by Fitting et al. [22] to be $R_{\mathrm{el}} \sim \rho^{-0.8} E_{\mathrm{el}}^{13}$ ( $R_{\mathrm{el}}$ is the radius of the electron range, $\rho$ the density of the absorber material and $E_{\mathrm{el}}$ the electron energy). This approximation [20] is reasonably good for energies well above the K-edge of the material. In the case of $4 \mathrm{keV}$ incident $\mathrm{X}$-rays, the charge cloud radius is on the order of $300 \mathrm{~nm}$ for backilluminated pnCCDs. In a detector with a depleted volume depth of $500 \mu \mathrm{m}$ and bias voltages around $-200 \mathrm{~V}$, electrons need about $5 \mathrm{~ns}$ to reach the potential minimum on the front side. During this time, electrostatic repulsion and diffusion widen the electron distribution to approximately $7 \mu \mathrm{m}$ (rms). As X-rays with energies below $4 \mathrm{keV}$ are absorbed within $10 \mu \mathrm{m}$, i.e. close to the entrance (back-) side, the generated holes are more rapidly taken out a

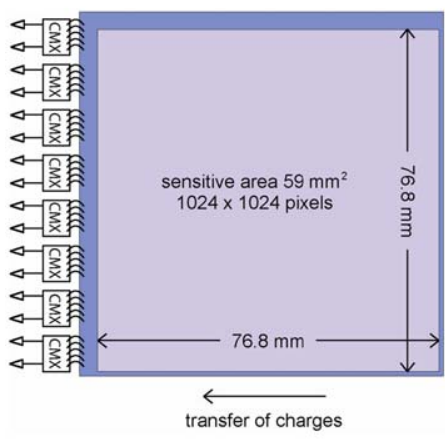

b

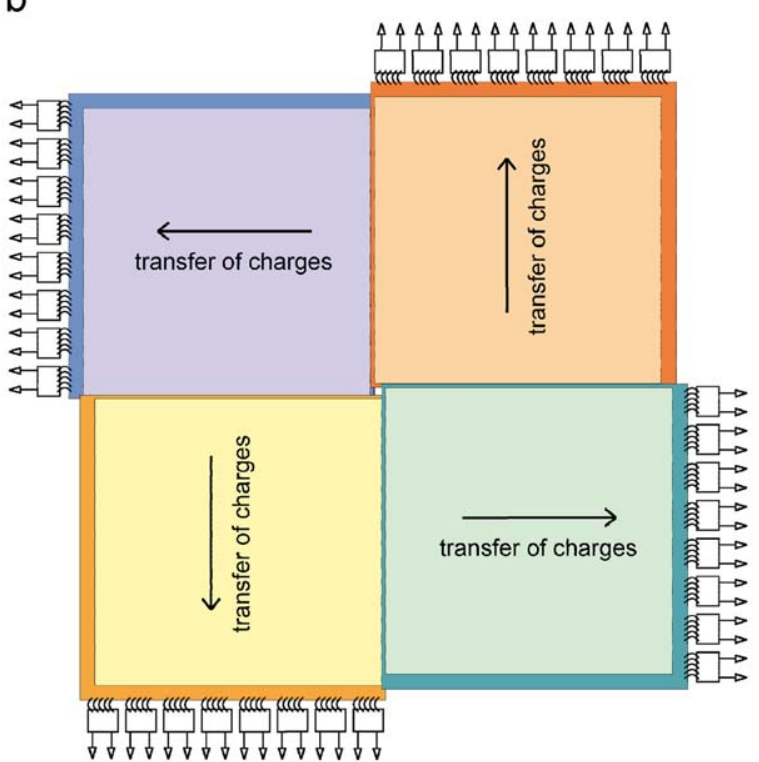

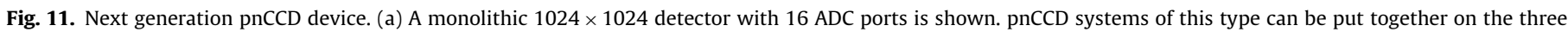

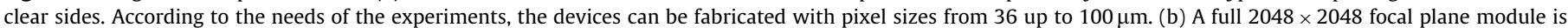
shown. It contains less than $1 \%$ of insensitive gaps, and a total area of $240 \mathrm{~cm}^{2}$ can be read out in less than $8 \mathrm{~ms}$. 
through the negatively biased back contact. In cases where many $\mathrm{X}$-rays, e.g. several thousands, hit the detector at the same time and position, the individual X-rays will be absorbed according to the $1 / e$ attenuation law in point-like interactions along a line with individual but heavily overlapping charge spheres. For energies below $4 \mathrm{keV}$ the length of such an "X-ray track" in silicon will be up to $10 \mu \mathrm{m}$ long and the local charge clouds of the individual X-rays get mixed, leading to a charge "tube" with high charge densities of electrons and holes. Then, in a first step, the electric field of the detector removes only the outer skin of charges of the plasma while the inner part stays neutral but highly conductive-and expands further by diffusion. This is why a cloud with a high density of electrons expands more than extrapolated from low densities.

We have started a project to calculate the time-dependent charge cloud expansion and are developing a three dimensional device simulator solving the Poisson and continuity equations with a finite volume method on the basis of the given pnCCD geometry, under realistic operating conditions and doping concentrations. In this calculation, up to 800,000 electrons were homogeneously deposited in a sphere of $10 \mu \mathrm{m}$ diameter with the outer radius $2 \mu \mathrm{m}$ above the backside of the depleted volume. The electrons were exposed to the internal electrical fields of the detector. In Fig. 12, the expanded electron distribution is shown after $1.3 \mathrm{~ns}$ in one quarter of a full pixel $\left(75 \times 75 \times 150 \mu \mathrm{m}^{3}\right)$ with a footprint of $37.5 \times 37.5 \mu \mathrm{m}^{2}$. For technical reasons, it was not possible to simulate a larger volume with the required number of discrete elements. Moreover, we have
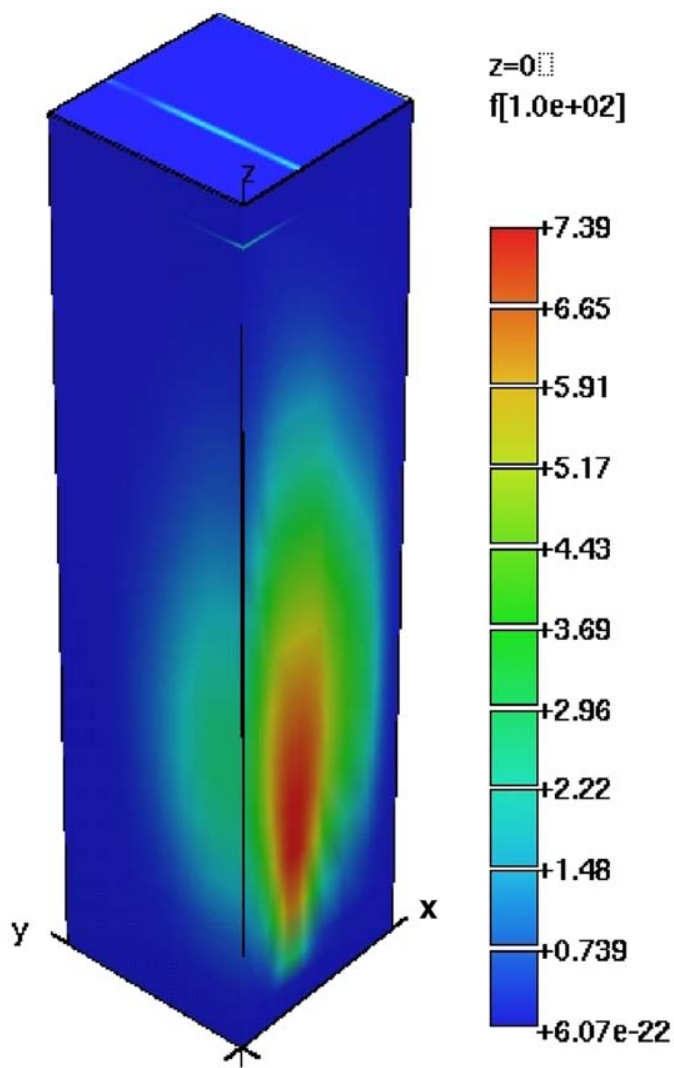

Fig. 12. Results of a charge cloud calculation using a numerical three-dimensional time-dependent model. The distribution of electrons is shown in colour coded units of the intrinsic charge carrier density $n_{i}=1.4 \times 10^{10}$ per $\mathrm{cm}^{3}$. The footprint in $x$ and $y$ is $37.5 \times 37.5 \mu \mathrm{m}^{2}$, in $z$ it is $150 \mu \mathrm{m}$. The full expansion of the electrons is prevented by the non-optimal boundary conditions of the partial, non-linear differential (van Roosbroeck equations). $1.3 \mathrm{~ns}$ after its generation, the original charge cloud with an initial diameter of $5 \mu \mathrm{m}$ has expanded to a size of $25 \mu \mathrm{m} \times 65 \mu \mathrm{m}$. A study which solves the symmetry related cloud compression is underway. assumed Neumann's boundary conditions at all vertical faces of the pixels. This artificially confines the signal charges inside the pixel as it assumes an infinite array of pixels and clouds. Under these conditions and with the approximations mentioned, the width of the charge cloud upon arrival in the potential minimum is on the order of half the pixel size. In the future, these model calculations will be further developed (e.g. enlarging the volume) with the goal to come to a reliable simulation of the time-dependent charge cloud expansion which should result in a simplification of the basic semiconductor structures and, thus, finally save computer resources.

An experimental approach to obtain information on the charge cloud expansion is the irradiation of the pnCCD entrance window with alpha particles from an ${ }^{241} \mathrm{Am}$ source with $\alpha$-energies around $5.5 \mathrm{MeV}$. They produce $1.6 \times 10^{6}$ electron-hole pairs, about $10^{6}$ of them at the end of the $28 \mu \mathrm{m}$ track in a volume of a few tens of $\mu \mathrm{m}^{3}$. The spatial full-width at half-maximum of the measured charge distribution was approximately $150 \mu \mathrm{m}$.

Both the simulations and measurements confirm that more detailed detector modeling is required to fully characterize the response as a function of the incident X-ray energy, intensity and spatial distribution. In order to ensure observation of weak signals in the immediate neighborhood of a strong, intense peak, an understanding of the electron dynamics in the detector is of utmost importance.

\section{Experiments at BESSY and FLASH}

Before integrating the pnCCD into a more complex experimental end station and in order to gain operational experience at light sources, a variety of tests were performed by (a) using medium to high energy polychromatic X-rays $(5-35 \mathrm{keV}$, white beam) at the synchrotron BESSY and (b) low-energy, pulsed FEL $\mathrm{X}$-rays from 30 up to $180 \mathrm{eV}$ energy at the FLASH free electron laser, DESY. The measurements were performed between 2007 and 2009. For both experiments, we have used the pnCCD type shown in Fig. 13. The chips have a format of $256 \times 256$ in the

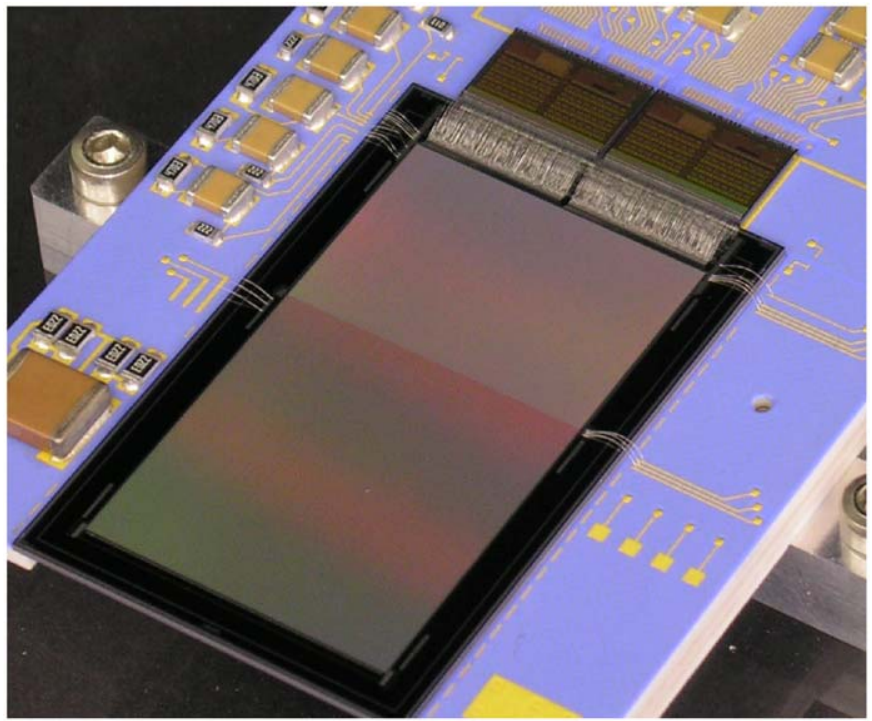

Fig. 13. pnCCD system with $256 \times 512$ pixels used in the experiments at FLASH and BESSY. The pnCCD is the large chip on the U-shaped blue ceramic board. The CAMEX ASICs on the upper right are wire bonded to the pnCCDs on-chip JFET amplifier. The pitch between the bonds is $71 \mu \mathrm{m}$. The sensitive area for X-rays is $3.7 \mathrm{~cm}^{2}$ in the imaging area and $2.5 \mathrm{~cm}^{2}$ in the frame store area. For the BESSY experiments, the system was operated with $200 \mathrm{~Hz}$ frame rate in a single photon counting spectroscopy mode. At FLASH, it was operated at 5 and $10 \mathrm{~Hz}$ according to the FEL bunch structure. 
image area (pixel size $75 \times 75 \mu \mathrm{m}^{2}, A=3.7 \mathrm{~cm}^{2}$ ) and the same format but smaller pixels $\left(75 \times 51 \mu \mathrm{m}^{2}, A=2.5 \mathrm{~cm}^{2}\right)$ in the frame store area. These experiments at BESSY and FLASH covered the photon energy range, the read-out frame rate and the anticipated noise figures of the pnCCD system to demonstrate the single photon detection ability over the full energy bandwidth under realistic experimental conditions. The pioneering measurements at FLASH did not allow testing the full dynamic range of the devices with X-rays nor the effects of the charge cloud expansion due to low intensities of scattered light. In these single shot cluster experiments, a maximum of 10 X-rays of $90 \mathrm{eV}$ were detected per pixel. Therefore, these tests will be extended in dedicated measurements at FLASH and LCLS in the near future.

\subsection{Experiments at BESSY}

The experiment at BESSY was performed within a spectral range of $5 \mathrm{keV}<E<25 \mathrm{keV}$ with a maximum photon flux of the order of $10^{10} \mathrm{ph} / \mathrm{s}$ at $\sim 7 \mathrm{keV}$ (beam size $2 \times 0.1 \mathrm{~mm}^{2}$ ) [23]. As a specimen, we used a Cd-stearate multilayer mirror with vertical lattice spacing of $D=5 \mathrm{~nm}$, illuminated under a fixed angle $\left(\varphi=0.8^{\circ}\right)$ of incidence with respect to the sample surface. The detector was placed at a distance of $98 \mathrm{~cm}$ from the mirror at a position containing the specularly reflected beam spot. In order to guarantee both the full energy selectivity and sufficient statistical significance, the reflected beam was attenuated by thin aluminum foils to an integrated intensity below $10^{5}$ counts/s. The signal was continuously recorded for a few minutes with a read-out speed of 200 frames per second, corresponding to typical repetition rates at LCLS or SCSS. It was the aim of the experiment to simultaneously record position and energy of the incident X-rays. Therefore, we needed to make sure that any pixel on the pnCCD is hit only once during the $5 \mathrm{~ms}$ integration time of a single frame. After integration of many frames, the data can be presented as 2D frames $(X, Y$ spatial coordinates) representing a plane perpendicular to the direction of the reflected beam stacked along a third dimension, i.e. the energy, $E$ (see Fig. 14) [22]. In such a representation, each $2 \mathrm{D}$ frame corresponds to a single GrazingIncidence Small-Angle X-ray Scattering (GISAXS) image as it is typically recorded in monochromatic experiments without energy dispersive detection.

As can be seen clearly in the insets in Fig. 14, which show the extension along the $X$ and $Y$ directions of the photons for different energies, the $X$-dimension is limited by the lateral beam size of $2 \mathrm{~mm}$. The intensity band along $Y$ contains the specularly reflected beam at the reflection angle and diffusely scattered intensity whenever $\alpha_{f}$ fulfills the Bragg condition $\alpha_{f}=6.4 /(2 \cdots D E) . \alpha_{i}$ denotes the incident angle, $\alpha_{f}$ the outgoing angle with respect to the scattering plane. Additional spots seen in the front plane stem from Bragg reflection of the 2D lattice of the $\mathrm{Cd}$ ions. The planes $(X, E)$ and $(Y, E)$ show a series of Bragg peaks lined up along the $E$-axis at $Y$ and $X=0$, respectively. The Bragg peak intensity is extended along the direction oblique to the $E$-axis representing the diffuse-resonant scattering originating from the correlated interface roughness of the multilayer.

In a conventional experiment using monochromatic radiation, the $(X, E)$ 2D map is typically recorded by a series of consecutive scans of $\alpha_{f}$ for fixed $\alpha_{i}$. Similarly, the $(Y, E)$ map requires variation of an angle perpendicular to the incident plane for fixed $\alpha_{f}$ or $\alpha_{i}$. Obviously, any line scan can be extracted from the 3D data field when the 2D spatial images are taken as a function of the photon energy and using white incoming light. As an example, a line scan of specular reflectivity is presented in Fig. 15. It clearly shows two multilayer Bragg peaks and additional Kiessig fringes with higher oscillation frequency, which provides a measure of the total

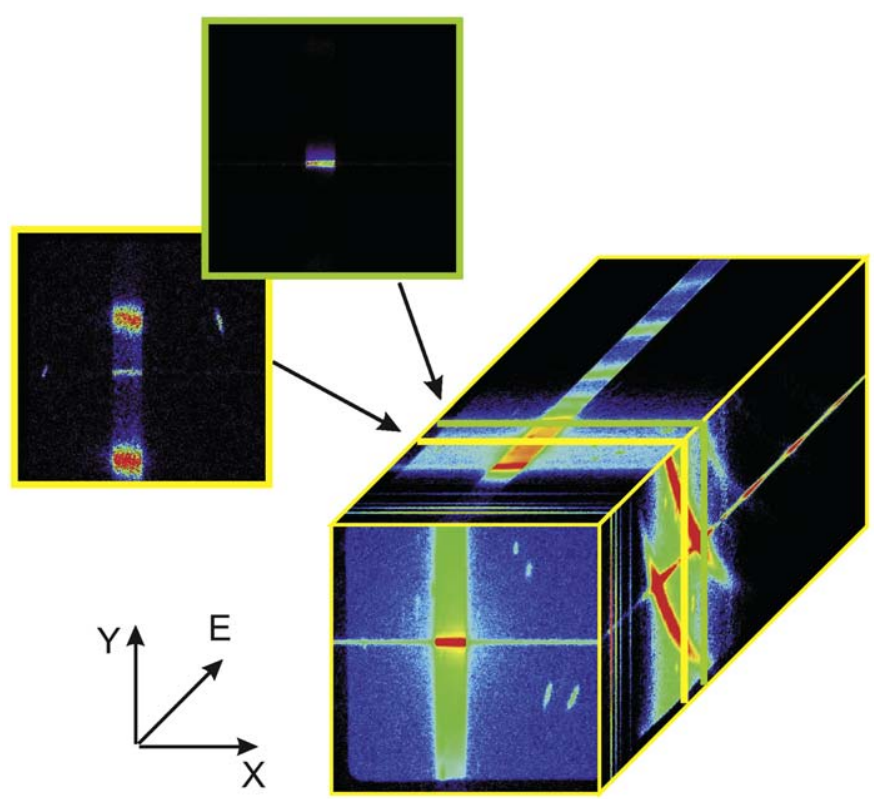

Fig. 14. 3-D representation of the energy-dispersive reflectivity of a Cd-stearate multilayer mirror using the pnCCD detector (see text). The mirror was illuminated by white light from a bending magnet. The displayed area in $x$ and $y$ is the $256 \times 256$ image area of the pnCCD with a size of $3.7 \mathrm{~cm}^{2}$. The energy scale starts at $7 \mathrm{keV}$ and ends at $35 \mathrm{keV}$. The broad green vertical line is due to the continuous flux of X-rays, which were hitting the pnCCD during the transfer of the image towards the readout nodes. The number of photons in the central peak (red spot) is typically 100 times higher than in the out-of-time band (green).

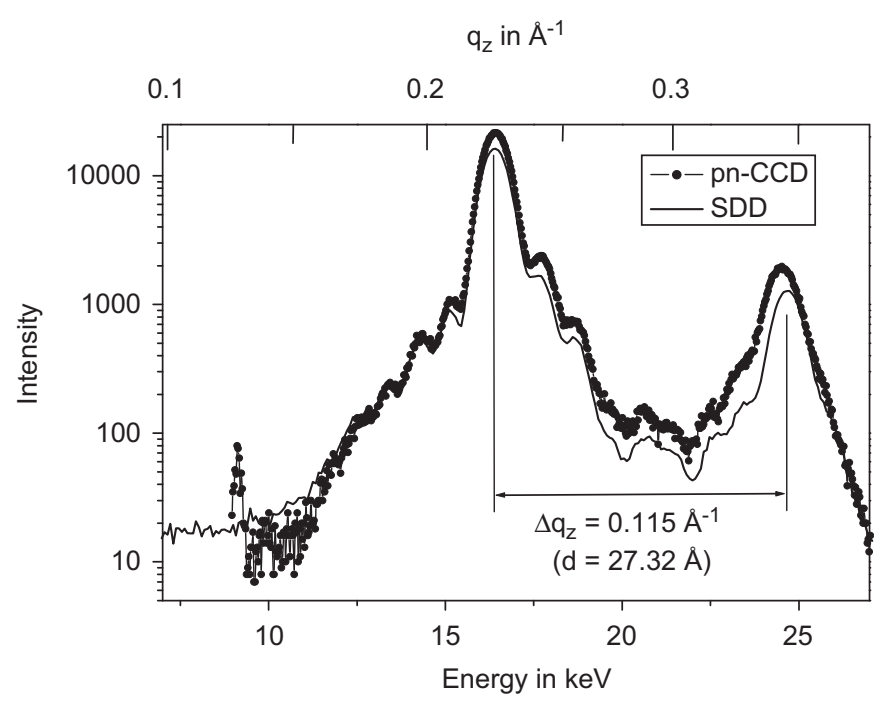

Fig. 15. Energy line scan along the specular direction extracted from the 3-D data shown in Fig. 14. Data from the pnCCD (filled circles) are compared to the results obtained with an energy dispersive point detector (line denoted by "SDD"; see text). The detector systems were not cross calibrated. For details see [22].

thickness of the multilayer film [8]. For comparison, we have also plotted a scan recorded by an energy-dispersive point detector (Röntec XFlash) under the same geometric conditions and which is denoted by the solid line (SDD) in the figure. The additional, upper scale in Fig. 15 represents the reciprocal space coordinate $q_{z}$ corresponding to the real space coordinate $E$ in the lower scale. Obviously, the results obtained by both detectors are very similar and only differ in the relative intensity at the higher 
photon energies. The data evaluation in terms of $q$-space and further experimental details has been published in [23].

In summary, the test measurements at the BESSY synchrotron clearly prove the single photon counting, energy-dispersive capabilities of our detector that were recorded at a frame readout speed matching the repetition rates at the LCLS and SCSS. Moreover, the present results impressively underline the advantage of using a pnCCD for such kind of experiments. In essence, the pnCCD combines $256 \times 256$ independent energy-dispersive point detectors which give a clear advantage for white-beam experiments if the energy resolution of the pnCCD is sufficient for the process under investigation.

\subsection{Experiments at FLASH}

As mentioned in the introduction, intense, short-wavelength femtosecond light pulses from free electron lasers are expected to open the door to a wide variety of investigations with unprecedented spatial and temporal resolution [24]. Along these lines, nano-sized clusters emerging from a supersonic jet were irradiated at FLASH with about $10^{12}$ photons per shot, focused to a spot size of a few microns, corresponding to power densities exceeding $10^{14} \mathrm{~W} / \mathrm{cm}^{2}$. Under these conditions, the sample in the laser focus is completely ionized on a femtosecond time scale [25]. This initiates a whole series of secondary effects such as ultra-fast electron migration, Coulomb explosion [26] and plasma recombination [27], potentially accompanied by the emission of fluorescence radiation, which has yet to be observed. As outlined in the introduction, imaging of nanostructures [28] requires that their sub-nanometer order is not obscured or destroyed by the FEL beam within the timescale necessary for image detection. Gaining insight into the underlying ultra-short, non-linear dynamic processes in the short wavelength, intense laser regime through single shot photon scattering experiments is of utmost importance for reaching this goal.

For scattering experiments on clusters, the pnCCD can be operated either in the high-dynamic-range or the single-photoncounting mode with photon energy resolution. The latter is ideal for the current purposes because it allows the distinction between scattered light and fluorescence photons from plasma recombination processes.

In Fig. 16 the single photon counting properties of a pnCCD is illustrated in an energy spectrum recorded for a series of cluster scattering events for $90 \mathrm{eV}$ incoming FLASH photons. It displays a clearly resolved photon line with a full-width half-maximum (FWHM) of the distribution of only $38.9 \mathrm{eV}$, an excellent value close to the theoretical limit, given the system readout noise of 2.5 electrons (rms)

The theoretical limit given by the Fano ionization statistics and the noise floor of 2.5 electrons (rms) is $\Delta E=28 \mathrm{eV}$ (FWHM). The difference to the measured value of $\Delta E=38 \mathrm{eV}$ (FWHM) arises from the setting of the noise threshold of $3 \sigma$, i.e. 7.5 electrons in this specific case. Split and partial events from the incoming $\mathrm{X}$-rays ( $90 \mathrm{eV}$ generate typically 25 electron-hole pairs) lead to a pixel content below threshold and therefore widen the FWHM towards the lower energies.

A small contribution of "signal pile-up" events at double the photon energy can be observed (in a logarithmic representation only), resulting from the detection of two photons hitting one pixel before it was read out. Event patterns in which the total energy was distributed in two neighboring pixels were recombined to one X-ray event. The peak at lower energies in Fig. 16 represents the read-out noise, which is partly suppressed via a threshold discriminator at $28 \mathrm{eV}$, corresponding to a 3- $\sigma$ cut on the noise floor.

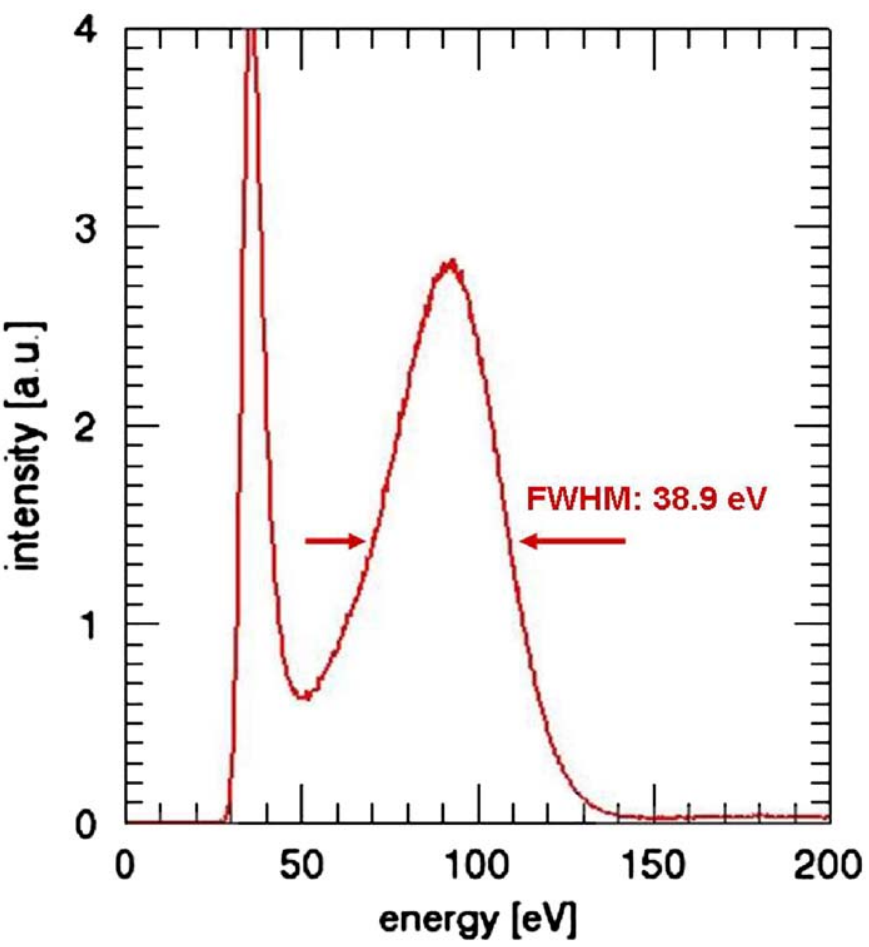

Fig. 16. Energy resolution measured at FLASH with $90 \mathrm{eV}$ VUV photons. Every photon generates approximately 25 electron-hole pairs. They have been detected with a read noise of 2.5 electrons (rms). The measured FWHM is only $38.9 \mathrm{eV}$. The plot contains all events: signal pile-up as well as pattern pile-up and split events. Events hitting two neighboring pixels have been recombined to one entry in the spectrum.

The data show that the main intensity of the detected photons is due to scattered light and that fluorescence light or signal from charged particles was not significant. In future experiments in the $\mathrm{X}$-ray spectral regime, we expect that the distinction between scattered and fluorescent photons may be a key feature for obtaining clean images for further analysis. Moreover, the yield of fluorescence photons will provide information on dynamic processes occurring during the irradiation and an appropriate experiment of this nature has been proposed to be performed at the LCLS by our collaboration. Last-but-not-least, the energy resolution of the pnCCD detector may open the door for applying novel two-color pump-probe strategies using high-harmonic radiation contributions contained in the FEL beam.

Fig. 17 shows single-shot detector images of Xe clusters with a radius of about $50 \mathrm{~nm}$. The data were recorded at a wavelength of $13.5 \mathrm{~nm}$, a pulse length of $10 \mathrm{fs}$, and a power density of about $10^{14} \mathrm{~W} / \mathrm{cm}^{2}$, which was reached by back-reflecting and focusing the FEL beam with a spherical multilayer mirror onto the cluster target. The compact design of our test-pnCCDs with an insensitive area of only $3 \mathrm{~mm}$ at the edge allowed positioning them in immediate proximity of the incoming/exiting light beam, allowing coverage of scattering angles from about $10^{\circ}$ to $30^{\circ}$. Here, the pnCCD directly faces the interaction region and is mounted at a distance of about $70 \mathrm{~mm}$, perpendicular to the polarization plane of the FEL. In total, three pnCCDs were installed in the experiment, covering angles from $10^{\circ}$ to $80^{\circ}$. In Fig. 17a, a single cluster was in the focus of the FEL pulse giving rise to a regular Mie diffraction pattern. Fig. 17b depicts a situation in which one of the clusters is in the shadow of a second one, such that interference phenomena overlie the scattering pattern. Note that this is a situation where one of the clusters (the properties of which would have to be exactly known) might be viewed as 

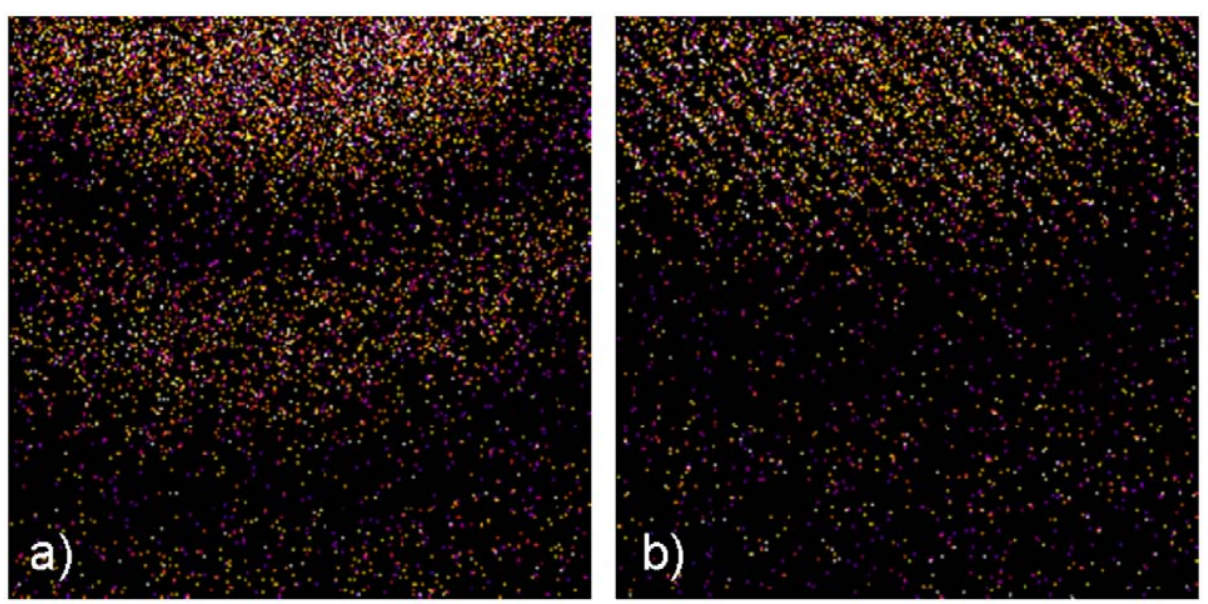

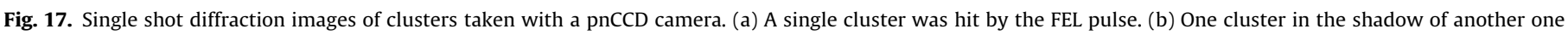

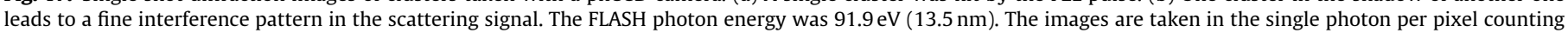
limit, allowing spectral analysis of each pixel with the resolution shown in Fig. 17.

emitting a reference wave for irradiating the second one, thus, generating essentially a holographic image of it. This scenario will be exploited in future experiments.

Importantly, the present measurements indicate that even weakly bound van-der-Waals systems "stay intact" for the duration of the pulse insofar as the position of the atoms and their overall extension is concerned. Detailed inspection of the intensity of the diffraction rings covering a larger area obtained when the three pnCCDs were in place, however, also clearly shows that the refractive index of the clusters has changed significantly during the pulse. This provides a first direct hint on the ultra-fast, fewfemtosecond electron dynamics initiated by the FEL light. Since in any experiment with sub-nanometer resolution the short-wave length, keV energy FEL radiation is mainly absorbed by inner-shell electrons with cross sections exceeding those for elastic scattering by at least one order of magnitude at $10 \mathrm{keV}$, the ultra-fast rearrangement of the electrons might pose serious challenges. Indeed, present scenarios assume that the FEL pulse has to be shorter than $10 \mathrm{fs}$, the typical life time of a carbon K-shell hole against Auger-decay, in order to suppress effectively its decay setting free another electron from the L-shell. Under conditions that are assumed to be suited for "single-molecule imaging", namely $10^{13}$ photons focused to a spot of $100 \mathrm{~nm}$ diameter, K-shell electrons are removed at an average rate of about one per 100 attoseconds. Thus, the second K-shell electron will be immediately removed as well, and, if the pulse is longer than the Auger life-time, the "refilled" $\mathrm{K}$-shell electron is also removed, resulting in the delocalization of at least four electrons from each carbon atom within less than $10 \mathrm{fs}$ (taking shake-off processes not into account).

Our present results provide a first glimpse on such ultra-fast electronic response functions in nano-scaled, mesoscopic objects in terms of a modified refractive index that has to be used to explain the experimental data. In general, this impressively underlines the unique information that can be retrieved from single shot, energy and angular dispersive photon diffraction and fluorescence measurements, which will be indispensable to approach the final goal of single shot molecule imaging. The present data also clearly prove that the pnCCDs can be safely and reliably operated under typical FEL and single particle imaging conditions.

\section{Conclusion}

We have planned, designed and assembled a novel multipurpose, highly flexible end station, the CFEL-ASG Multi-Purpose
(CAMP) chamber, for a large variety of applications at upcoming free electron lasers spanning the VUV to the hard X-ray wavelength regimes. The CAMP chamber accommodates a unique combination of various state-of-the-art imaging detector concepts. Two broad-range, energy dispersive ( $50 \mathrm{eV}-25 \mathrm{keV})$, singlephoton-counting and large area pnCCD pixel detector systems are flexibly mounted together with improved, high-resolution reaction microscope or velocity map imaging spectrometer devices for coincident multi-dimensional electron and ion momentum imaging. In its fully equipped operation mode, this allows, for the first time, simultaneous detection of angular- and energy(momentum-resolved) electrons, ions and scattered or fluorescent photons. From these correlated (in some cases, even coincident) and time-dependent many-particle data, we expect to obtain complete information on the dynamic processes occurring when ultra-intense FEL pulses at X-ray photon energies interact with matter, with single atoms and ions, with simple to complex molecules, with clusters and other nano-particles, with solid matter, surfaces or plasmas-all of which are a completely unexplored terra incognita of light-matter interaction.

Details of the chambers, the outline, design and mounting of the different targets and detectors, partly to be completed in subsequent phases, have been provided. Moreover, the results of extensive tests of the pnCCDs obtained at the BESSY synchrotron as well as at FLASH have been presented and demonstrate their capabilities for future experiments at X-ray free electron laser sources. This system will be employed in the first round of experiments at the LCLS in November 2009.

\section{Acknowledgements}

Support by central funds of the Max-Planck Society for advancing the science with FEL sources within the Advanced Study Group at the CFEL and previous support within the "Innovation Fund" of the President is gratefully acknowledged. Production and testing of the pnCCD devices would have been impossible without the excellent work of the staff of MPI semiconductor laboratory, the help of the DESY staff during beamtimes. We gratefully acknowledge the competent technical support of André Hömke and Carlo Schmidt from the MPG-ASG during the design and construction of CAMP chamber. The project was partially supported by BMBF no 05KS7KT1 and 05KS7PSA and Virtuelles Institut grant VH-VI-302. MPI semiconductor laboratory acknowledges the support of the Johannes-Heidenhain- 
Stiftung. Funding from the European XFEL for the simulation studies of the charge spreading is gratefully acknowledged.

\section{References}

[1] V. Ayvazyan, et al., Eur. Phys. Lett. 100 (2008) 133401; W. Ackermann, et al., Nat. Photonics 1 (2007) 336.

[2] LCLS Conceptual Design Report, SLAC-R-593, April 2002, UC-414

[3] T. Shintake, et al., Nat. Photonics 2 (2008) 555

[4] M. Altarelli, et al. (Eds.), European XFEL Technical Design Report (DESY 2006097, Hamburg, Germany, 2006), ISBN:978-3-935702-17-1.

[5] M. Porro, et al., NSS Conference Record, IEEE, 19-25 October 2008, pp. 1578-1586.

[6] R. Moshammer, et al., Nucl. Instr. and Meth. B 108 (1996) 425; J. Ullrich, et al., Rep. Prog. Phys. 66 (2003) 223202

[7] A.T. Eppink, D.H. Parker, Rev. Sci. Instrum. (1997) 3477.

[8] T. Geue, et al., J. Chem. Phys. 110 (1999) 8104.

[9] O. Jagutzki, et al., IEEE Trans. Nucl. Sci. NS-49 (2002) 2477.
[10] H. Fukuzawa, et al., Phys. Rev. A 79 (2009) 031201.

[11] M. Kurka, et al., J. Phys. B: At. Mol. Opt. Phys. 42 (2009) 141002.

[12] R. Moshammer, et al., Phys. Rev. Lett. 98 (2007) 203001.

[13] A. Rudenko, et al., Phys. Rev. Lett. 101 (2008) 073003.

[14] Y.H. Jiang, et al., Phys. Rev. Lett. 102 (2009) 123002.

[15] A. Vredenborg, et al., Rev. Sci. Instrum. 79 (2008) 063108.

[16] P. Johnsson, et al., J. Mod. Opt. 55 (2008) 2693.

[17] D. Rolles, et al., Nucl. Instr. and Meth. B 261 (2007) 170.

[18] L. Strüder, et al., Rev. Sci. Instrum. 68 (1997) 4271.

[19] L. Strüder, et al., Astron. Astrophys. 365 (2001) 18.

[20] L. Strüder, et al., Nucl. Instr. and Meth. A 288 (1990) 227.

[21] S. Herrmann, et al., NSS conference record '07, vol. 3, 2007, p. 2398.

[22] H.J. Fitting, Phys. Status Solidi (a) 26 (1974) 525.

[23] W. Leitenberger, et al., J. Synchrotron. Rad. 15 (2008) 449.

[24] C. Bostedt, et al., Nucl. Instr. and Meth. A 601 (2009) 108.

[25] C. Bostedt, et al., Phys. Rev. Lett. 100 (2008) 133401.

[26] H. Thomas, et al., J. Phys. B: At. Mol. Opt. Phys. 42 (2009) 134018.

[27] M. Hoener, et al., J. Phys. B: At. Mol. Opt. Phys. 41 (2008) 181001.

[28] H. Chapman, et al., Nature 448 (2007) 676. 